Article

\title{
Synthesis of (1,2,3-triazol-4-yl)methyl Phosphinates and (1,2,3-Triazol-4-yl)methyl Phosphates by Copper-Catalyzed Azide-Alkyne Cycloaddition
}

\author{
Anna Tripolszky ${ }^{1}$, Krisztina Németh ${ }^{2}$, Pál Tamás Szabó ${ }^{2}$ and Erika Bálint ${ }^{1, *(1)}$ \\ 1 Department of Organic Chemistry and Technology, Budapest University of Technology and Economics, \\ 1521 Budapest, Hungary; tripolszky.anna@mail.bme.hu \\ 2 MS Metabolomics Laboratory, Instrumentation Center, Research Centre for Natural Sciences, \\ Hungarian Academy of Sciences, Magyar Tudósok Krt. 2., H-1117 Budapest, Hungary; \\ nemeth.krisztina.94@ttk.mta.hu (K.N.); szabo.pal@ttk.mta.hu (P.T.S.) \\ * Correspondence: ebalint@mail.bme.hu; Tel.: +36-1-463-3653
}

Received: 21 May 2019; Accepted: 30 May 2019; Published: 31 May 2019

\begin{abstract}
An efficient and practical method was developed for the synthesis of new (1,2,3-triazol-4-yl)methyl phosphinates and (1,2,3-triazol-4-yl)methyl phosphates by the copper(I)-catalyzed azide-alkyne cycloaddition (CuAAC) of organic azides and prop-2-ynyl phosphinate or prop-2-ynyl phosphate. The synthesis of (1-benzyl-1H-1,2,3-triazol-4-yl)methyl diphenylphosphinate was optimized with respect to the reaction parameters, such as the temperature, reaction time, and catalyst loading. The approach was applied to a range of organic azides, which confirmed the wide scope and the substituent tolerance of the process. The method elaborated represents a novel approach for the synthesis of the target compounds.
\end{abstract}

Keywords: 1,2,3-triazol; triazolylmethyl phosphinate; triazolylmethyl phosphate; copper-catalyzed azide-alkyne cycloaddition; click reaction

\section{Introduction}

The family of 1,2,3-triazoles has attracted considerable attention in the last decades due to the wide range of their applications in medicine and biochemistry, as well as in materials science [1-3]. Several 1,2,3-triazoles proved to be effective antibacterial, antifungal, anticancer, antiviral, or anti-inflammatory agents [4]. The 1,4-disubstituted $1 H$-1,2,3-triazoles also have important applications as agrochemicals, photostabilizers, dyes, or anticorrosives [5-8].

Organophosphorus compounds also present significant importance as biologically active agents [9]. The 1,2,3-Triazolyl phosphonates combine the advantages of the triazole and the phosphonate moieties [10]. Additionally, several triazolylphosphonate derivatives were found to be suitable for bioconjugation [11,12] or showed an anti-HIV effect [13].

The most convenient synthetic method for the preparation of 1,4-disubstituted 1H-1,2,3-triazoles is the $\mathrm{Cu}(\mathrm{I})$-catalyzed 1,3-dipolar (Huisgen) cycloaddition of azides with alkynes (CuAAC) (the "click reaction"), which was developed by Meldal and Sharpless [14,15]. The 1,2,3-Triazolyl phosphonates may be synthesized by the reaction of azides with phosphorus-containing acetylenes [12, 13,16-20] or by the cycloaddition of azides incorporating a phosphonate moiety with alkynes [21-24]. In this paper, reports of the former method will be presented in detail.

The 1,3-dipolar cycloaddition of benzyl azide and ethyl ethynylphosphonate (1) was carried out in the absence of any catalyst at reflux temperature in toluene (Scheme 1) [16]. The reaction was not selective, two regioisomers, 1,2,3-triazolyl-4-phosphonate (2a) and 1,2,3-triazolyl-5-phosphonate (2b) were formed. 


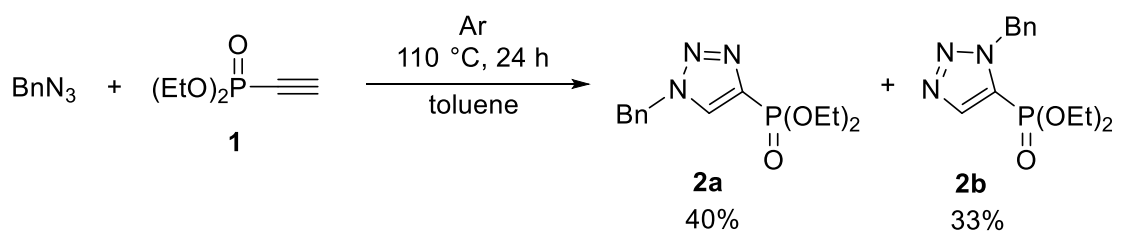

Scheme 1. Cycloaddition of benzyl azide and diethyl ethynylphosphonate (1).

Bisphosphonates incorporating a triazole ring (4) were prepared by the CuAAC reaction of a propargyl-substituted bisphosphonate (3) and organic azides (Scheme 2) [17,18]. The reactions were performed using different methods. According to method A, copper iodide was applied as a catalyst in the presence of $\mathrm{N}, \mathrm{N}$-diisopropylethylamine (DIPEA) as a base in tetrahydrofuran (THF) [17]. In another case, the $\mathrm{Cu}(\mathrm{I})$ catalyst was formed in situ from copper(II) sulfate pentahydrate and sodium ascorbate in the mixture of tert-butanol and water [18].

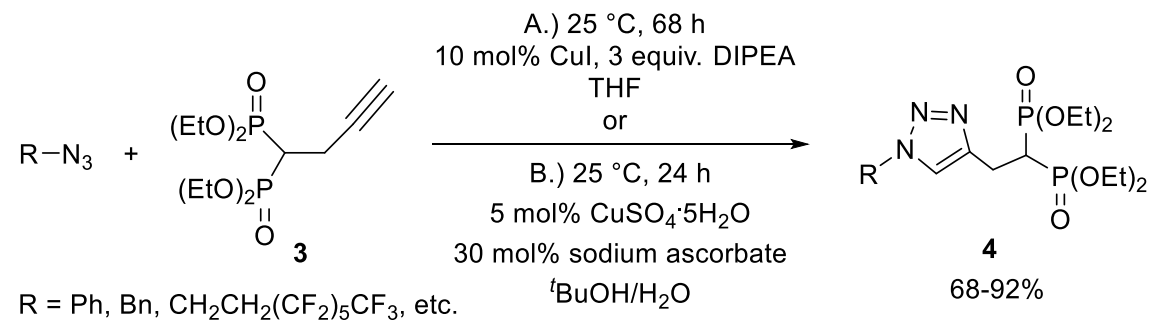

Scheme 2. Synthesis of bisphosphonates bearing a triazole ring (4) via CuAAc reaction.

Röschenthaler and co-workers performed the synthesis of triazole-containing $\alpha-\mathrm{CF}_{3}-\alpha$-aminophosphonates (6) by the $\mathrm{Cu}(\mathrm{I})$-catalyzed cycloaddition of azides and ethynylor propargyl-substituted phosphonate (5) (Scheme 3) [19].

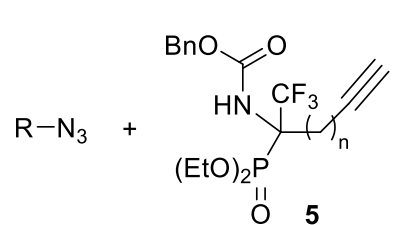

$\mathrm{R}=\mathrm{Ph}, \mathrm{CH}_{2} \mathrm{CH}_{2}\left(\mathrm{CF}_{2}\right)_{7} \mathrm{CF}_{3}$, etc. $\mathrm{n}=0,1$ $25^{\circ} \mathrm{C}, 8-12 \mathrm{~h}$

$5 \mathrm{~mol} \% \mathrm{CuSO}_{4} \cdot 5 \mathrm{H}_{2} \mathrm{O}$

$30 \mathrm{~mol} \%$ sodium ascorbate

${ }^{t} \mathrm{BuOH} / \mathrm{H}_{2} \mathrm{O}$

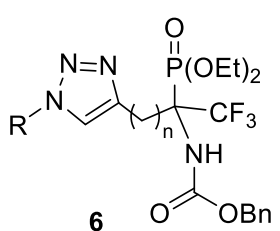

$38-92 \%$

Scheme 3. Synthesis of triazole-containing $\alpha-\mathrm{CF}_{3}-\alpha$-aminophosphonates (6).

The click reaction of 2-azidoethanol and triprop-2-ynyl phosphate (7) was also reported, in which a triazole-functionalized phosphate flame-retardant monomer (8) was synthesized (Scheme 4) [20].

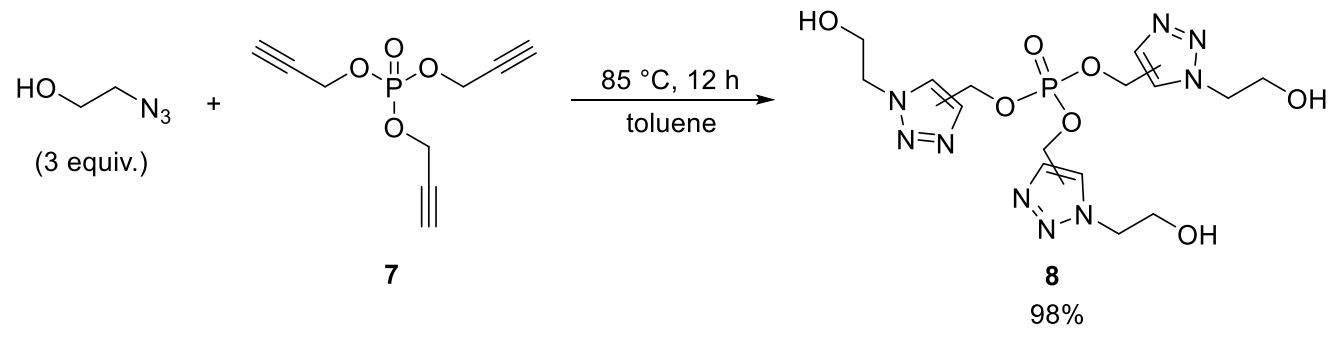

Scheme 4. Synthesis of a triazole-functionalized phosphate monomer (8).

To the best of our knowledge, there is no example for the azide-alkyne cycloaddition of simple azides and prop-2-ynyl phosphinate or diethyl prop-2-ynyl phosphate. The reaction of prop-2-ynyl 
diphenylphosphinate was reported only with steroidal azides [25]. Hence, we set a sight on building a phosphinate or phosphate side-chain on position 4 of the triazole ring by the click reaction with simple azides.

In this paper, we report on an efficient and fast synthesis of (1,2,3-triazol-4-yl)methyl phosphinates and (1,2,3-triazol-4-yl)methyl phosphates by the copper(I)-catalyzed 1,3-dipolar (Huisgen) cycloaddition of organic azides and prop-2-ynyl phosphinates or diethyl prop-2-ynyl phosphate.

\section{Results and Discussion}

At first, the starting materials of the cycloadditions were prepared. The synthesis of azides was carried out based on the literature data $[26,27]$ (Scheme 5). The benzyl or substituted benzyl bromides were reacted with 1.5 equivalents of sodium azide at room temperature for $24 \mathrm{~h}$ in a mixture of acetone/water in a ratio of $4: 1$, and the corresponding azides (9a-f) were obtained in yields of $80-93 \%$ (Scheme 5/I, Method A). The synthesis of octyl-, $i$-octyl- and cyclohexyl azides (9g-i) was performed using 1.2 equivalents of sodium azide at $70^{\circ} \mathrm{C}$ in dimethylformamide (DMF) (Scheme 5/I, Method B). For the preparation of phenyl azide (11), aniline was reacted with sodium nitrite in $\mathrm{HCl} / \mathrm{H}_{2} \mathrm{O}$ solution at $0{ }^{\circ} \mathrm{C}$ for $15 \mathrm{~min}$, and the diazonium salt (10) formed was further reacted with sodium azide at ambient temperature (Scheme 5/II). After workup, phenyl azide (11) was obtained in a yield of 65\%.

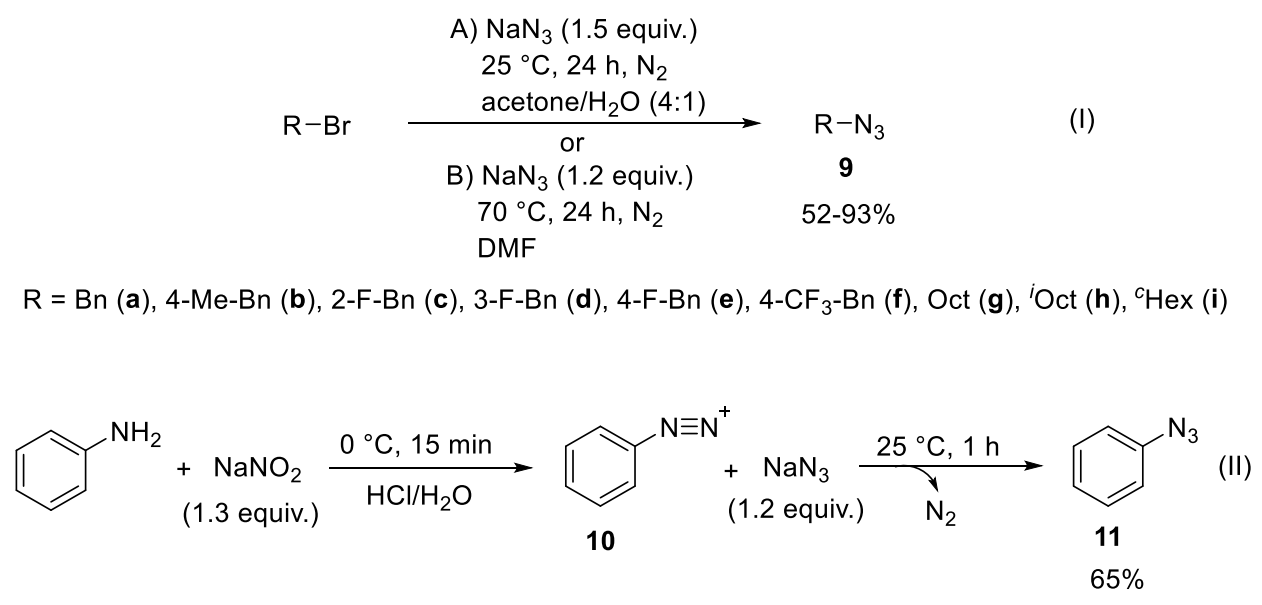

Scheme 5. Synthesis of organic azides (9a-i and 11).

The synthesis of the prop-2-ynyl diphenylphosphinate (12a) and the diethyl prop-2-ynyl phosphate (12b) was carried out by the reaction of diphenylphosphinic chloride or diethyl chlorophosphate with propargyl alcohol in the presence of triethylamine in diethyl ether (Scheme 6). The phosphorus-containing alkynes (12a and $\mathbf{1 2} \mathbf{b}$ ) were isolated in yields of $88 \%$ and $72 \%$, respectively.

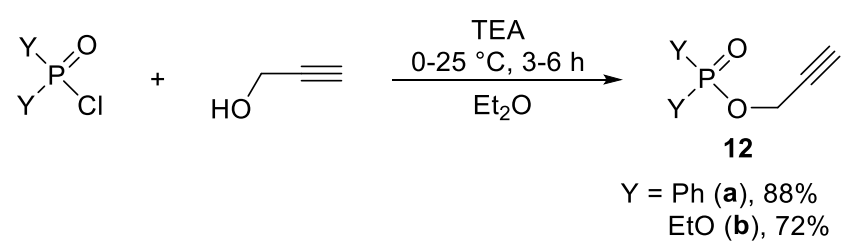

Scheme 6. Synthesis of prop-2-ynyl phosphinate (12a) and diethyl prop-2-ynyl phosphate (12d).

As the next step of our work, the 1,3-dipolar cycloaddition of benzyl azide (9a) and prop-2-ynyl diphenylphosphinate (12a) was investigated in the presence of copper(II) sulfate pentahydrate and sodium ascorbate in the mixture of tert-butanol/water (4:1) (Table 1). Performing the reaction at room temperature using $5 \mathrm{~mol} \%$ of $\mathrm{CuSO}_{4} \cdot 5 \mathrm{H}_{2} \mathrm{O}$ and $30 \mathrm{~mol} \%$ of sodium ascorbate, it was complete after $1 \mathrm{~h}$ and, after column chromatography, the desired (1-benzyl-1H-1,2,3-triazol-4-yl)methyl 
diphenylphosphinate (13a) was obtained in a yield of 84\% (Table 1, Entry 1). Decreasing the amount of the reducing agent (sodium ascorbate) to $10 \mathrm{~mol} \%$, the conversion was only $78 \%$ under the conditions applied before $\left(25^{\circ} \mathrm{C}, 60 \mathrm{~min}\right)$ (Table 1, Entry 2$)$. In this case, complete conversion could be reached after $3 \mathrm{~h}$ (Table 1, Entry 3). Carrying out the reaction at $60^{\circ} \mathrm{C}$ for $5 \mathrm{~min}$ under conventional heating, the triazolylmethyl phosphinate (13a) was formed in a conversion of 93\% (Table 1, Entry 4). Applying microwave (MW) irradiation, the result obtained was similar (Table 1, Entry 5), thus further experiments were performed in an oil bath. Increasing the reaction time to $10 \mathrm{~min}$, the cycloaddition was complete and product 13a was isolated in a yield of $89 \%$ (Table 1, Entry 6). In the next step, the effect of the catalyst loading was studied (Table 1/Entries 7-9). Using $3 \%$ of $\mathrm{CuSO}_{4} \cdot 5 \mathrm{H}_{2} \mathrm{O}$ and $5 \mathrm{~mol} \%$ of sodium ascorbate, the reaction was similar to the previous experiment (Table 1, Entries 6 and 7). Upon decreasing the amount of the $\mathrm{Cu}(\mathrm{II})$ catalyst to $2 \%$, the conversion was lower (90\%) (Table 1, Entry 7). Based on the results obtained, a temperature of $60{ }^{\circ} \mathrm{C}$, a reaction time of $10 \mathrm{~min}$ and application of $3 \mathrm{~mol} \%$ of $\mathrm{CuSO}_{4} \cdot 5 \mathrm{H}_{2} \mathrm{O}$, as well as $5 \mathrm{~mol} \%$ of sodium ascorbate, were found to be the optimum parameters (Table 1, Entry 7).

Table 1. Optimization of the reaction of benzyl azide (9a) and propynyl diphenylphosphinate (11a).

\begin{tabular}{|c|c|c|c|c|c|c|c|}
\hline & $9 a$ & $12 a$ & H & $\begin{array}{c}\mathrm{T}, \mathrm{t} \\
\mathrm{CuSO}_{4} \cdot 5 \mathrm{H}_{2} \mathrm{O} \\
\text { odium ascorbat } \\
\mathrm{BuOH} / \mathrm{H}_{2} \mathrm{O}(4: 1\end{array}$ & & $=\mathrm{N} \quad \mathrm{O}^{\prime} \mathrm{O}^{\prime}{ }^{\prime} \mathrm{Ph}$ & \\
\hline \multirow{2}{*}{ Entry } & \multirow{2}{*}{ Mode of Heating } & \multirow{2}{*}{$\mathrm{T}\left[{ }^{\circ} \mathrm{C}\right]$} & \multirow{2}{*}{$t$ [min] } & \multicolumn{2}{|c|}{ Catalyst [mol\%] } & \multirow{2}{*}{ Conversion $[\%]^{a}$} & \multirow{2}{*}{ Yield $[\%]^{b}$} \\
\hline & & & & $\mathrm{CuSO}_{4} \cdot 5 \mathrm{H}_{2} \mathrm{O}$ & Sodium Ascorbate & & \\
\hline 1 & - & 25 & 60 & 5 & 30 & 100 & 84 \\
\hline 2 & - & 25 & 60 & 5 & 10 & 78 & - \\
\hline 3 & - & 25 & 180 & 5 & 10 & 100 & 89 \\
\hline 4 & $\Delta$ & 60 & 5 & 5 & 10 & 93 & - \\
\hline 5 & MW & 60 & 5 & 5 & 10 & 92 & - \\
\hline 6 & $\Delta$ & 60 & 10 & 5 & 10 & 100 & 89 \\
\hline 7 & $\Delta$ & 60 & 10 & 3 & 5 & 100 & 91 \\
\hline 8 & $\Delta$ & 60 & 10 & 2 & 5 & 90 & - \\
\hline
\end{tabular}

${ }^{\text {a }}$ Based on ${ }^{31}$ P NMR (phosphorus-31 nuclear magnetic resonance); ${ }^{b}$ Isolated yield; MW (microwave).

In the next series of experiments, the cycloaddition of prop-2-ynyl diphenylphosphinate (12a) with a wide range of organic azides (9 and 11) was studied under the optimized conditions (Scheme 7). The reactions were complete in all cases. Using 4-methylbenzyl azide (9b), the [1-(4-methylbenzyl)-1H-1,2,3-triazol-4-y])methyl diphenylphosphinate (13b) was isolated in a yield of $86 \%$. Changing for fluoro-substituted benzyl azides (2-, 3- or 4-fluorobenzyl azide) (9c-e), the desired triazolylmethyl phosphinates (13c-e) were obtained in yields of $81-83 \%$ after column chromatography. Carrying out the reaction starting from 4-(trifluoromethyl)benzyl azide (9f), the product $\mathbf{1 3 f}$ was prepared in a yield of $88 \%$. Applying octyl azide (9g), the corresponding (1-octyl-1H-1,2,3-triazol-4-yl)methyl diphenylphosphinate $\mathbf{( 1 3 g )}$ was obtained in a yield of $89 \%$, while using iso-octyl azide (9h), product $\mathbf{1 3 h}$ was isolated in a yield of $77 \%$. The cyclohexyl azide (9i) was also tried out as the azide component; however, the triazolylmethyl phosphinate (13i) could be obtained in a slightly lower yield (62\%) due to the steric effects of the cyclohexyl group. Finally, the click reaction of phenyl azide (11) was performed, and the desired triazolylmethyl phosphinate (13j) was synthesized in a yield of $82 \%$. 


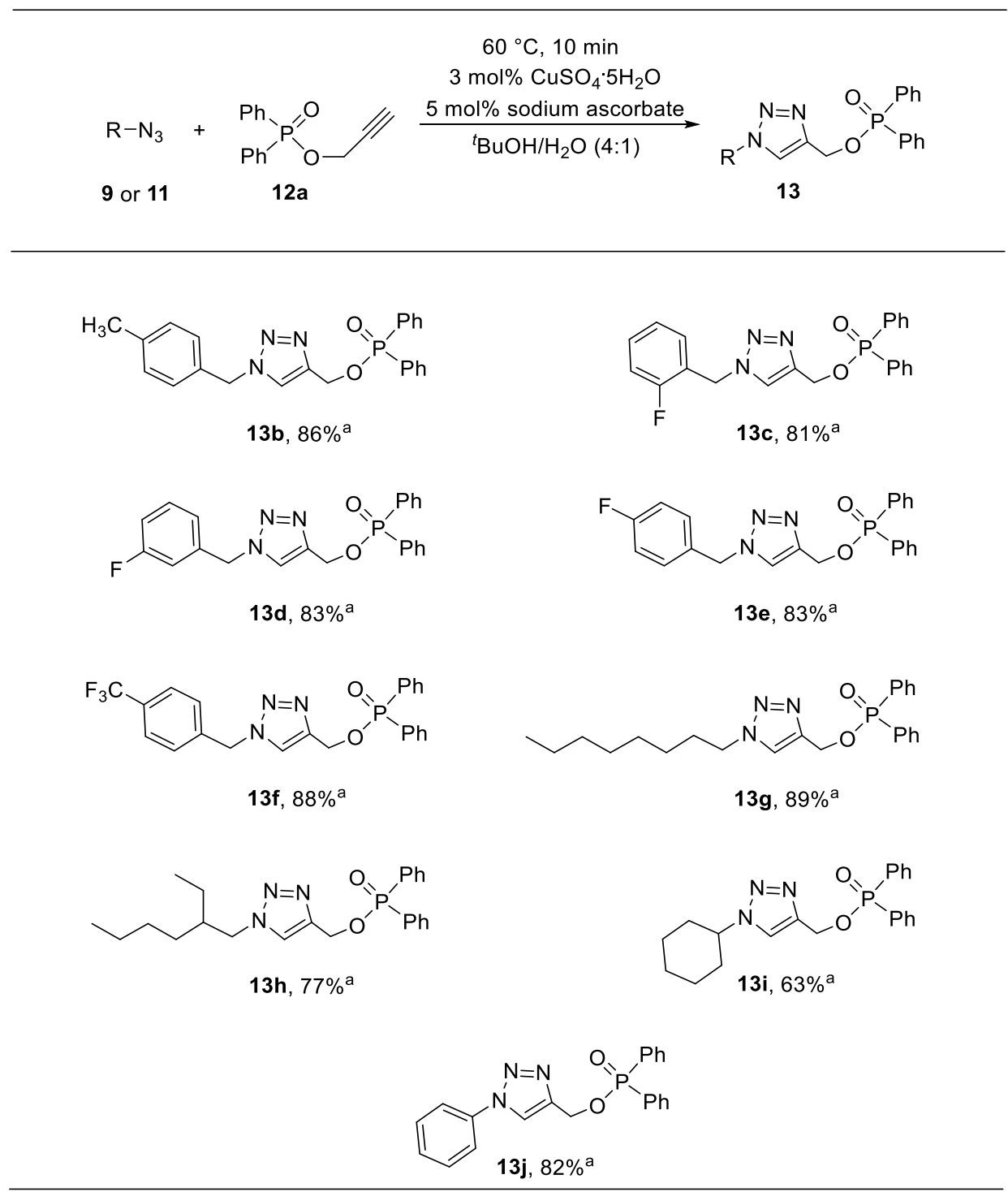

${ }^{a}$ Isolated yield.

Scheme 7. Synthesis of (1H-1,2,3-triazol-4-yl)methyl diphenylphosphinates (13).

The cycloaddition of benzyl azide (9a) and diethyl prop-2-ynyl phosphate (12b) was also investigated (Table 2). Using the optimized conditions ( $3 \mathrm{~mol} \%$ of $\mathrm{CuSO}_{4} \cdot 5 \mathrm{H}_{2} \mathrm{O}$ and $5 \mathrm{~mol} \%$ of sodium ascorbate, $60^{\circ} \mathrm{C}, 10 \mathrm{~min}$ ), the reaction was incomplete, and the (1-benzyl-1H-1,2,3-triazol-4-yl)methyl diethyl phosphate (14a) was formed in a conversion of only $48 \%$ (Table 2, Entry 1). Increasing the reaction time to $20 \mathrm{~min}$, the conversion increased to $59 \%$; however, after $30 \mathrm{~min}$, the reaction was complete, and the triazolylmethyl phosphate (14a) was obtained in a yield of 75\% (Table 2, Entries 2 and 3). The diethyl prop-2-ynyl phosphate (12b) proved to be somewhat less reactive in the click reaction as compared to the prop-2-ynyl diphenylphosphinate (12a).

In the next round, the reaction of diethyl prop-2-ynyl phosphate (12b) was also carried out with a wide range of organic azides (9 and 11) in the presence of $3 \mathrm{~mol} \%$ of $\mathrm{CuSO}_{4} \cdot 5 \mathrm{H}_{2} \mathrm{O}$ and $5 \mathrm{~mol} \%$ of sodium ascorbate at $60^{\circ} \mathrm{C}$ for $30 \mathrm{~min}$ (Scheme 8). Applying substituted benzyl azides (4-methyl-, 2-, 3- or 4-fluorobenzylazide and 4-(trifluoromethyl)benzyl azide) (11b-f), the corresponding (1-benzyl-1H-1,2,3-triazol-4-yl)methyl diethyl phosphate derivatives (14b-f) were prepared in yields of $54-69 \%$. Performing the cycloaddition starting from octyl or iso-octyl azide (11g or $\mathbf{1 1 h}$ ), the desired products $(14 \mathrm{~g}$ or $14 \mathrm{~h}$ ) were isolated in yields of $60 \%$ and $54 \%$, respectively. The reaction of cyclohexyl azide (11i) and diethyl prop-2-ynyl phosphate (12b) was also carried out, and the product $14 \mathbf{i}$ was 
obtained in a yield of $51 \%$. Using aromatic azide, such as phenyl azide (11), the triazolylmethyl phosphate (14j) was synthesized in a yield of $77 \%$.

Table 2. Reaction of benzyl azide (9a) and diethyl prop-2-ynyl phosphate (12b).

$60^{\circ} \mathrm{C}, \mathrm{t}$

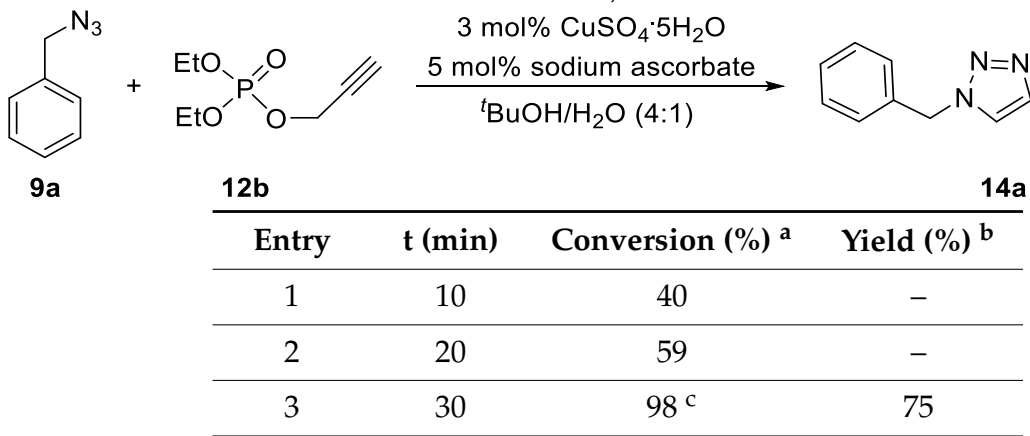

${ }^{a}$ Based on ${ }^{31} \mathrm{P}$ NMR. ${ }^{\mathrm{b}}$ Isolated yield. ${ }^{\mathrm{c}}$ No change for longer reaction time.

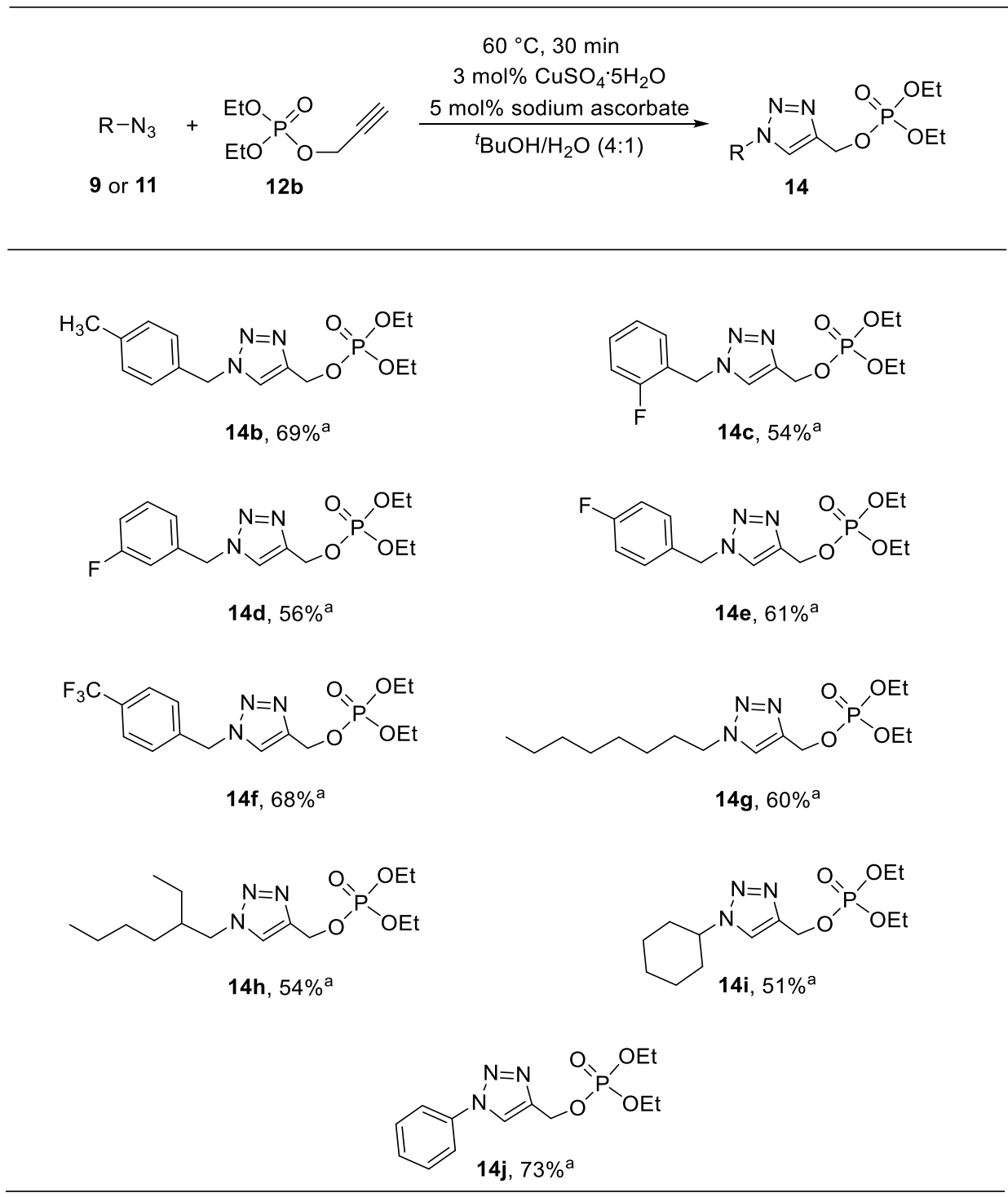

a Isolated yield.

Scheme 8. Synthesis of (1H-1,2,3-triazol-4-yl)methyl diethyl phosphates (14). 


\section{Materials and Methods}

\subsection{General}

The reactions under conventional heating were carried out in an oil bath. The microwave-assisted experiments were performed in a $300 \mathrm{~W}$ CEM Discover focused microwave reactor (CEM Microwave Technology Ltd., Buckingham, UK) equipped with a pressure controller using 5-10 W irradiation under isothermal conditions.

High-performance liquid chromatography-mass spectrometry (HPLC-MS) measurements were performed with an Agilent 1200 liquid chromatography system coupled with a 6130 quadrupole mass spectrometer equipped with an ESI ion source (Agilent Technologies, Palo Alto, CA, USA). Analysis was performed at $40{ }^{\circ} \mathrm{C}$ on a Gemini C18 column $(150 \mathrm{~mm} \times 4.6 \mathrm{~mm}, 3 \mu \mathrm{m}$; Phenomenex, Torrance, CA, USA) with a mobile phase flow rate of $0.6 \mathrm{~mL} / \mathrm{min}$. The composition of eluent A was $0.1 \%\left(\mathrm{NH}_{4}\right)(\mathrm{HCOO})$ in water; eluent $\mathrm{B}$ was $0.1 \%\left(\mathrm{NH}_{4}\right)(\mathrm{HCOO})$ and $8 \%$ water in acetonitrile, $0-3 \mathrm{~min}$ $5 \%$ B, 3-13 min gradient, 13-20 min 100\% B. The injection volume was $5 \mu \mathrm{L}$. The chromatographic profile was registered at $254 \mathrm{~nm}$. The MSD operating parameters were as follows: positive ionization mode, scan spectra from m/z 120 to 1200 , drying gas temperature $300{ }^{\circ} \mathrm{C}$, nitrogen flow rate $10 \mathrm{~L} / \mathrm{min}$, nebulizer pressure 60 psi, capillary voltage $4000 \mathrm{~V}$.

High-resolution mass spectrometric measurements were performed using a Sciex 5600+ Q-TOF (time-of-flight) mass spectrometer in positive electrospray mode.

The ${ }^{31} \mathrm{P},{ }^{1} \mathrm{H},{ }^{13} \mathrm{C}$, NMR spectra were taken in $\mathrm{CDCl}_{3}$ solution on a Bruker AV-300 spectrometer (Bruker AXS GmBH, Karlsruhe, Germany) operating at 121.5, 75.5, and $300 \mathrm{MHz}$, respectively. Chemical shifts are downfield relative to $85 \% \mathrm{H}_{3} \mathrm{PO}_{4}$ and TMS (spectra for all compounds synthesized can be found in Supplementary).

\subsection{General Procedure for the Synthesis of Benzyl Azides (Method A)}

To a stirred solution of $10.0 \mathrm{mmol}$ alkyl halides $(1.19 \mathrm{~mL}$ of benzyl bromide, $1.85 \mathrm{~g}$ of 4 -methylbenzyl bromide, $1.21 \mathrm{~mL}$ of 2-fluorobenzyl bromide, $1.23 \mathrm{~mL}$ of 3-fluorobenzyl bromide, $1.25 \mathrm{~mL}$ of 4-fluorobenzyl bromide, or $1.55 \mathrm{~mL}$ of 4-(trifluoromethyl)benzyl bromide) in $100 \mathrm{~mL}$ of acetone/ $\mathrm{H}_{2} \mathrm{O}$ 4:1 $(v / v)$ was added $15.0 \mathrm{mmol}(0.98 \mathrm{~g})$ of sodium azide. The reaction mixture was stirred at room temperature for $24 \mathrm{~h}$. After, the reaction was extracted with $\mathrm{Et}_{2} \mathrm{O}(3 \times 50 \mathrm{~mL})$, dried over $\mathrm{Na}_{2} \mathrm{SO}_{4}$, and concentrated under reduced pressure to give benzyl azides as pale yellow oils. The following azides were thus prepared (Table 3):

Table 3. MS Data for benzyl azides (9a-f).

\begin{tabular}{cccc}
\hline Compound & Yield & {$[\mathbf{M + H}]^{+}$found } & [M+H $^{+}$requires \\
\hline Benzyl azide (9a) [26] & $93 \%(1.24 \mathrm{~g})$ & 134.0725 & 134.0718 \\
4-Methylbenzyl azide (9b) [28] & $80 \%(1.18 \mathrm{~g})$ & 148.0880 & 148.0874 \\
2-Fluorobenzyl azide (9c) [29] & $68 \%(1.02 \mathrm{~g})$ & 152.0632 & 152.0624 \\
3-Fluorobenzyl azide (9d) [30] & $76 \%(1.14 \mathrm{~g})$ & 152.0633 & 152.0624 \\
4-Fluorobenzyl azide (9e) [28] & $83 \%(1.25 \mathrm{~g})$ & 152.0632 & 152.0624 \\
4-(Trifluoromethyl)benzyl azide (9f) [31] & $86 \%(1.72 \mathrm{~g})$ & 202.0601 & 202.0592 \\
\hline
\end{tabular}

\subsection{General Procedure for the Synthesis of Alkyl Azides (Method B)}

To a stirred solution of $10.0 \mathrm{mmol}$ alkyl halides $(1.76 \mathrm{~mL}$ of octyl bromide, $1.78 \mathrm{~mL}$ of $i$ so-octyl bromide, or $1.23 \mathrm{~mL}$ of bromocyclohexane) in $20 \mathrm{~mL}$ of DMF, $12.0 \mathrm{mmol}$ ( $0.78 \mathrm{~g}$ ) of sodium azide was added. The reaction mixture was stirred at $70{ }^{\circ} \mathrm{C}$ for $24 \mathrm{~h}$ in an oil bath. After, the reaction was extracted with $\mathrm{Et}_{2} \mathrm{O}(3 \times 50 \mathrm{~mL})$, dried over $\mathrm{Na}_{2} \mathrm{SO}_{4}$, and concentrated under reduced pressure to give alkyl azides as pale yellow oils. The following azides were thus prepared (Table 4): 
Table 4. MS Data for alkyl azides $(9 \mathrm{~g}-\mathbf{i})$.

\begin{tabular}{cccc}
\hline Compound & Yield & [M+H $^{+}{ }_{\text {found }}$ & {$[\mathbf{M + H}]^{+}$requires } \\
\hline Octyl azide (9g) [32] & $67 \%(1.04 \mathrm{~g})$ & 156.1514 & 156.1501 \\
Iso-octyl azide (9h) [33] & $55 \%(0.85 \mathrm{~g})$ & 156.1514 & 156.1501 \\
Cyclohexyl azide (9i) [27] & $52 \%(0.65 \mathrm{~g})$ & 126.1038 & 126.1031 \\
\hline
\end{tabular}

\subsection{General Procedure for the Synthesis of Phenyl Azide}

To a stirred solution of $0.46 \mathrm{~mL}$ aniline $(5.0 \mathrm{mmol})$ in $25 \mathrm{~mL} 17 \% \mathrm{HCl}$ solution at $0{ }^{\circ} \mathrm{C}, 0.51 \mathrm{~g}$ $(7.5 \mathrm{mmol})$ of sodium nitrite in water $(3 \mathrm{~mL})$ was added. After stirring for $15 \mathrm{~min}$, a solution of $0.32 \mathrm{~g}$ sodium azide $(7.5 \mathrm{mmol})$ in water $(3 \mathrm{~mL})$ was carefully added. The reaction was left to stir for $1 \mathrm{~h}$, followed by extraction with $\mathrm{Et}_{2} \mathrm{O}(3 \times 30 \mathrm{~mL})$. The combined organic layers were dried over $\mathrm{Na}_{2} \mathrm{SO}_{4}$, and carefully concentrated under reduced pressure to give phenyl azide as an orange oil.

Phenyl azide (11) [26]: Yield: 65\% (0.77 g) of compound 11 as orange oil; $[\mathrm{M}+\mathrm{H}]^{+}$found $=120.0567$, $\mathrm{C}_{6} \mathrm{H}_{6} \mathrm{~N}_{3}$ requires 120.0562 .

\subsection{General Procedure for the Synthesis of Prop-2-ynyl Diphenylphosphinate and Diethyl Prop-2-ynyl Phosphate}

To a stirred solution of $10 \mathrm{mmol}$ of diphenylphosphinic chloride $(1.91 \mathrm{~mL})$ or diethyl chlorophosphate $(1.44 \mathrm{~mL})$ in $10 \mathrm{~mL}$ of $\mathrm{Et}_{2} \mathrm{O}, 1.39 \mathrm{~mL}(10 \mathrm{mmol})$ of $\mathrm{Et}_{3} \mathrm{~N}$ and $0.50 \mathrm{~mL}(10.0 \mathrm{mmol})$ of propargyl alcohol at $0{ }^{\circ} \mathrm{C}$ were added under a nitrogen atmosphere. The solution was left stirring at room temperature for 3-6 h and the reaction mixture obtained was passed through a $1 \mathrm{~cm}$ silica gel layer using $\mathrm{Et}_{2} \mathrm{O}$. After evaporating the solvent, the products were obtained as white crystals (12a) or colorless oil (12b). The following products were thus prepared (Table 5):

Table 5. ${ }^{31} \mathrm{P}$ NMR and MS Data for prop-2-ynyl diphenylphosphinate (12a) and diethyl prop-2-ynyl phosphate (12b).

\begin{tabular}{cccccc}
\hline Compound & Yield & $\boldsymbol{\delta}_{\mathbf{P}}$ in $\mathbf{C D C l}_{\mathbf{3}}$ & $\boldsymbol{\delta}_{\mathbf{P}}[$ lit. $]$ in $\mathbf{C D C l}_{\mathbf{3}}$ & {$[\mathbf{M}+\mathbf{H}]^{+}$found } & {$[\mathbf{M}+\mathbf{H}]^{+}$requires } \\
\hline $\mathbf{1 2 a}$ & $88 \%(2.25 \mathrm{~g})$ & 34.3 & $34.2[25]$ & 257.0735 & 257.0731 \\
$\mathbf{1 2 b}$ & $72 \%(1.38 \mathrm{~g})$ & -0.4 & $-0.7[34]$ & 193.0639 & 193.0629 \\
\hline
\end{tabular}

\subsection{General Procedure for the Synthesis of (1H-1,2,3-Triazol-4-yl)methyl Phosphinates or Diethyl Phosphates}

The $1.0 \mathrm{mmol}$ organic azide (0.13 $\mathrm{g}$ of benzyl azide, $0.15 \mathrm{~g}$ of 4-methylbenzyl azide, $0.15 \mathrm{~g}$ of 2-fluorobenzyl azide, $0.15 \mathrm{~g}$ of 3-fluorobenzyl azide, $0.15 \mathrm{~g}$ of 4-fluorobenzyl azide, $0.20 \mathrm{~g}$ of 4-(trifluoromethyl)benzyl azide, $0.16 \mathrm{~g}$ of octyl azide, $0.16 \mathrm{~g}$ of iso-octyl azide, $0.13 \mathrm{~g}$ of cyclohexyl azide or $0.12 \mathrm{~g}$ of phenyl azide) and $1.0 \mathrm{mmol}$ acetylene ( $0.26 \mathrm{~g}$ of prop-2-ynyl diphenylphosphinate or $0.19 \mathrm{~g}$ of diethyl prop-2-ynyl phosphate) were suspended in a mixture of ${ }^{t} \mathrm{BuOH} / \mathrm{H}_{2} \mathrm{O}(4: 1)(2 \mathrm{~mL})$. To this $7.5 \mathrm{mg}(0.03 \mathrm{mmol})$ of $\mathrm{CuSO}_{4} \cdot 5 \mathrm{H}_{2} \mathrm{O}$ and $20 \mathrm{mg}(0.1 \mathrm{mmol})$ of sodium ascorbate were added. The mixture was stirred at $60^{\circ} \mathrm{C}$ for $10 \mathrm{~min}$. The resulting solution was extracted with ethyl acetate $(3 \times 30 \mathrm{~mL})$ and the combined organic layers were dried over $\mathrm{Na}_{2} \mathrm{SO}_{4}$. After evaporating the solvent, the crude product was purified by column chromatography using silica gel and dichloromethane/methanol 97:3 as the eluent. The following products were thus prepared:

(1-Benzyl-1H-1,2,3-triazol-4-yl)methyl diphenylphosphinate (13a): Yield: 91\% (0.35 g), white crystals; Mp: 91-92 ${ }^{\circ} \mathrm{C} ;{ }^{31} \mathrm{P} \mathrm{NMR}\left(\mathrm{CDCl}_{3}\right) \delta 33.5 ;{ }^{1} \mathrm{H}$ NMR $\left(\mathrm{CDCl}_{3}\right) \delta 5.18\left(\mathrm{~d},{ }^{3} \mathrm{~J}_{\mathrm{HP}}=9.1,2 \mathrm{H}, \mathrm{CH}_{2} \mathrm{O}\right), 5.46(\mathrm{~s}, 2 \mathrm{H}$, $\left.\mathrm{CH}_{2} \mathrm{Ph}\right), 7.20-7.27\left(\mathrm{~m}, 2 \mathrm{H}, \mathrm{C}_{2} \mathrm{H}\right), 7.32-7.37\left(\mathrm{~m}, 3 \mathrm{H}, \mathrm{C}_{3} \mathrm{H}, \mathrm{C}_{4} \mathrm{H}\right), 7.38-7.44\left(\mathrm{~m}, 4 \mathrm{H}, \mathrm{C}_{3^{\prime}} \mathrm{H}\right), 7.47-7.54(\mathrm{~m}$, $\left.2 \mathrm{H}, \mathrm{C}_{4}, \mathrm{H}\right), 7.52(\mathrm{~s}, 1 \mathrm{H}, \mathrm{CH}), 7.79\left(\mathrm{dd},{ }^{3} \mathrm{~J}_{\mathrm{HP}}=11.8,{ }^{3} \mathrm{~J}_{\mathrm{HH}}=7.4,4 \mathrm{H}, \mathrm{C}_{2}, \mathrm{H}\right) ;{ }^{13} \mathrm{C} \mathrm{NMR}\left(\mathrm{CDCl}_{3}\right) \delta 54.3$ $\left(\mathrm{CH}_{2} \mathrm{Ph}\right), 58.2\left(\mathrm{~d},{ }^{2} J_{\mathrm{CP}}=5.3, \mathrm{CH}_{2} \mathrm{O}\right), 123.8(\mathrm{CH}=), 128.3\left(\mathrm{C}_{2}\right), 128.7\left(\mathrm{~d},{ }^{3} J_{\mathrm{CP}}=13.3, \mathrm{C}_{3^{\prime}}\right), 128.8\left(\mathrm{C}_{4}\right), 129.3$ $\left(\mathrm{C}_{3}\right), 131.2\left(\mathrm{~d},{ }^{1} J_{\mathrm{CP}}=137.0, \mathrm{C}_{1^{\prime}}\right), 131.8\left(\mathrm{~d},{ }^{2} J_{\mathrm{CP}}=10.3, \mathrm{C}_{2^{\prime}}\right), 132.5\left(\mathrm{~d}, J_{\mathrm{CP}}=2.8, \mathrm{C}_{4^{\prime}}\right), 134.5\left(\mathrm{C}_{1}\right), 144.0$ $\left(\mathrm{d},{ }^{3} J_{\mathrm{CP}}=6.7, \mathrm{C}=\right) ;[\mathrm{M}+\mathrm{H}]^{+}$found $=390.1363 ., \mathrm{C}_{22} \mathrm{H}_{21} \mathrm{~N}_{3} \mathrm{O}_{2} \mathrm{P}$ requires 390.1371 . 
[1-(4-Methylbenzyl)-1H-1,2,3-triazol-4-yl]methyl diphenylphosphinate (13b): Yield: 86\% (0.34 g), white crystals; Mp: $119-121{ }^{\circ} \mathrm{C} ;{ }^{31} \mathrm{P}$ NMR $\left(\mathrm{CDCl}_{3}\right) \delta 33.3 ;{ }^{1} \mathrm{H}$ NMR $\left(\mathrm{CDCl}_{3}\right) \delta 2.35\left(\mathrm{~s}, 3 \mathrm{H}, \mathrm{CH}_{3} \mathrm{Ph}\right), 5.18(\mathrm{~d}$, $\left.{ }^{3} \mathrm{~J}_{\mathrm{HP}}=9.0,2 \mathrm{H}, \mathrm{CH}_{2} \mathrm{O}\right), 5.41\left(\mathrm{~s}, 2 \mathrm{H}, \mathrm{CH}_{2} \mathrm{Ph}\right), 7.07-7.24\left(\mathrm{~m}, 4 \mathrm{H}, \mathrm{C}_{3}, \mathrm{H}\right), 7.32-7.60\left(\mathrm{~m}, 7 \mathrm{H}, \mathrm{C}_{2} \mathrm{H}, \mathrm{C}_{3} \mathrm{H}, \mathrm{C}_{4}, \mathrm{H}\right)$, $7.79(\mathrm{~s}, 1 \mathrm{H}, \mathrm{CH}), 7.80\left(\mathrm{dd},{ }^{3} J_{\mathrm{HP}}=12.4,{ }^{3} \mathrm{~J}_{\mathrm{HH}}=7.4,4 \mathrm{H}, \mathrm{C}_{2}{ }^{\prime} \mathrm{H}\right) ;{ }^{13} \mathrm{C} \mathrm{NMR}\left(\mathrm{CDCl}_{3}\right) \delta 21.3\left(\mathrm{CH}_{3} \mathrm{Ph}\right), 54.2$ $\left(\mathrm{CH}_{2} \mathrm{Ph}\right), 58.2\left(\mathrm{~d},{ }^{2} J_{\mathrm{CP}}=5.3, \mathrm{CH}_{2} \mathrm{O}\right), 123.6(\mathrm{CH}=), 128.3\left(\mathrm{C}_{2}\right), 128.7\left(\mathrm{~d},{ }^{3} J_{\mathrm{CP}}=13.2, \mathrm{C}_{3^{\prime}}\right), 129.9\left(\mathrm{C}_{3}\right), 131.3$ $\left(\mathrm{d},{ }^{1} J_{\mathrm{CP}}=136.6, \mathrm{C}_{1^{\prime}}\right), 131.4\left(\mathrm{C}_{1}\right), 131.8\left(\mathrm{~d},{ }^{2} J_{\mathrm{CP}}=10.3, \mathrm{C}_{2^{\prime}}\right), 132.4\left(\mathrm{~d}, J_{\mathrm{CP}}=2.8, \mathrm{C}_{4^{\prime}}\right), 138.9\left(\mathrm{C}_{4}\right), 144.0(\mathrm{~d}$, $\left.{ }^{3} J_{\mathrm{CP}}=6.7, \mathrm{C}=\right) ;[\mathrm{M}+\mathrm{H}]^{+}$found $=404.1519, \mathrm{C}_{23} \mathrm{H}_{23} \mathrm{~N}_{3} \mathrm{O}_{2} \mathrm{P}$ requires 404.1527 .

[1-(2-Fluorobenzyl)-1H-1,2,3-triazol-4-yl]methyl diphenylphosphinate (13c): Yield: 81\% (0.33 g), pale yellow crystals; Mp: $91-93{ }^{\circ} \mathrm{C} ;{ }^{31} \mathrm{P}$ NMR $\left(\mathrm{CDCl}_{3}\right) \delta 33.5 ;{ }^{1} \mathrm{H}$ NMR $\left(\mathrm{CDCl}_{3}\right) \delta 5.18\left(\mathrm{~d},{ }^{3} J_{\mathrm{HP}}=8.9,2 \mathrm{H}\right.$, $\left.\mathrm{CH}_{2} \mathrm{O}\right), 5.52\left(\mathrm{~s}, 2 \mathrm{H}, \mathrm{CH}_{2} \mathrm{Ph}\right), 7.03-7.17\left(\mathrm{~m}, 2 \mathrm{H}, \mathrm{C}_{3} \mathrm{H}, \mathrm{C}_{5} \mathrm{H}\right), 7.18-7.26\left(\mathrm{~m}, 2 \mathrm{H}, \mathrm{C}_{6} \mathrm{H}\right), 7.28-7.53(\mathrm{~m}, 7 \mathrm{H}$, $\left.\mathrm{C}_{4} \mathrm{H}, \mathrm{C}_{3^{\prime}} \mathrm{H}, \mathrm{C}_{4^{\prime}} \mathrm{H}\right), 7.60(\mathrm{~s}, 1 \mathrm{H}, \mathrm{CH}), 7.79\left(\mathrm{dd},{ }^{3} J_{\mathrm{HP}}=12.3,{ }^{3} J_{\mathrm{HH}}=6.9,4 \mathrm{H}, \mathrm{C}_{2^{\prime}} \mathrm{H}\right) ;{ }^{13} \mathrm{C} \mathrm{NMR}\left(\mathrm{CDCl}_{3}\right) \delta$ $47.8\left(\mathrm{~d},{ }^{3} J_{\mathrm{CF}}=4.4, \mathrm{CH}_{2} \mathrm{Ph}\right), 58.1\left(\mathrm{~d},{ }^{2} J_{\mathrm{CP}}=5.3, \mathrm{CH}_{2} \mathrm{O}\right), 115.9\left(\mathrm{~d},{ }^{2} J_{\mathrm{CF}}=21.1, \mathrm{C}_{3}\right), 121.8\left(\mathrm{~d},{ }^{2} J_{\mathrm{CF}}=14.6\right.$, $\left.\mathrm{C}_{1}\right), 124.0(\mathrm{CH}=), 124.9\left(\mathrm{~d}, J_{\mathrm{CF}}=3.8, \mathrm{C}_{5}\right), 128.7\left(\mathrm{~d},{ }^{3} J_{\mathrm{CP}}=13.3, \mathrm{C}_{3^{\prime}}\right), 130.7\left(\mathrm{~d},{ }^{3} J_{\mathrm{CF}}=3.2, \mathrm{C}_{4}\right), 131.1(\mathrm{~d}$, $\left.{ }^{3} J_{\mathrm{CF}}=8.2, \mathrm{C}_{6}\right), 131.1\left(\mathrm{~d},{ }^{1} J_{\mathrm{CP}}=136.8, \mathrm{C}_{1^{\prime}}\right), 131.8\left(\mathrm{~d},{ }^{2} J_{\mathrm{CP}}=10.4, \mathrm{C}_{2^{\prime}}\right), 132.4\left(\mathrm{~d}, J_{\mathrm{CP}}=2.9, \mathrm{C}_{4^{\prime}}\right), 144.0(\mathrm{~d}$, $\left.{ }^{3} J_{\mathrm{CP}}=6.6, \mathrm{C}=\right), 160.0\left(\mathrm{~d},{ }^{1} J_{\mathrm{CF}}=248.1, \mathrm{C}_{2}\right) ;[\mathrm{M}+\mathrm{H}]^{+}$found $=408.1268, \mathrm{C}_{22} \mathrm{H}_{20} \mathrm{~N}_{3} \mathrm{O}_{2} \mathrm{FP}$ requires 408.1277.

[1-(3-Fluorobenzyl)-1H-1,2,3-triazol-4-yl]methyl diphenylphosphinate (13d): Yield: 83\% (0.34 g), pale yellow crystals; Mp: $93-94{ }^{\circ} \mathrm{C} ;{ }^{31} \mathrm{P} \mathrm{NMR}\left(\mathrm{CDCl}_{3}\right) \delta 33.7 ;{ }^{1} \mathrm{H} \mathrm{NMR}\left(\mathrm{CDCl}_{3}\right) \delta 5.20\left(\mathrm{~d},{ }^{3} \mathrm{~J}_{\mathrm{HP}}=9.3,2 \mathrm{H}\right.$, $\left.\mathrm{CH}_{2} \mathrm{O}\right), 5.46\left(\mathrm{~s}, 2 \mathrm{H}, \mathrm{CH}_{2} \mathrm{Ph}\right), 6.87-6.96\left(\mathrm{~m}, 1 \mathrm{H}, \mathrm{C}_{4} \mathrm{H}\right), 6.89-7.11\left(\mathrm{~m}, 2 \mathrm{H}, \mathrm{C}_{2} \mathrm{H}, \mathrm{C}_{6} \mathrm{H}\right), 7.26-7.36(\mathrm{~m}, 1 \mathrm{H}$, $\left.\mathrm{C}_{5} \mathrm{H}\right), 7.37-7.55\left(\mathrm{~m}, 6 \mathrm{H}, \mathrm{C}_{3}, \mathrm{H}, \mathrm{C}_{4}, \mathrm{H}\right), 7.59(\mathrm{~s}, 1 \mathrm{H}, \mathrm{CH}), 7.79\left(\mathrm{dd},{ }^{3} \mathrm{~J}_{\mathrm{HP}}=12.4,{ }^{3} \mathrm{~J}_{\mathrm{HH}}=6.8,4 \mathrm{H}, \mathrm{C}_{2}, \mathrm{H}\right) ;{ }^{13} \mathrm{C}$ $\operatorname{NMR}\left(\mathrm{CDCl}_{3}\right) \delta 53.6\left(\mathrm{~d}, J_{\mathrm{CF}}=2.0, \mathrm{CH}_{2} \mathrm{Ph}\right), 58.2\left(\mathrm{~d},{ }^{2} J_{\mathrm{CP}}=5.3, \mathrm{CH}_{2} \mathrm{O}\right), 115.2\left(\mathrm{~d},{ }^{2} J_{\mathrm{CF}}=22.2, \mathrm{C}_{4}\right), 116.0$ $\left(\mathrm{d},{ }^{2} J_{\mathrm{CF}}=21.0, \mathrm{C}_{2}\right), 123.8\left(\mathrm{~d}, J_{\mathrm{CF}}=3.1, \mathrm{C}_{6}\right), 123.9(\mathrm{CH}=), 128.7\left(\mathrm{~d},{ }^{3} J_{\mathrm{CP}}=13.3, \mathrm{C}_{3^{\prime}}\right), 130.9\left(\mathrm{~d},{ }^{3} J_{\mathrm{CF}}=8.3\right.$, $\left.\mathrm{C}_{5}\right), 131.1\left(\mathrm{~d},{ }^{1} J_{\mathrm{CP}}=136.7, \mathrm{C}_{1^{\prime}}\right), 131.8\left(\mathrm{~d},{ }^{2} J_{\mathrm{CP}}=10.3, \mathrm{C}_{2^{\prime}}\right), 132.5\left(\mathrm{~d}, J_{\mathrm{CP}}=2.8, \mathrm{C}_{4^{\prime}}\right), 136.8\left(\mathrm{~d},{ }^{3} J_{\mathrm{CF}}=7.4\right.$, $\left.\mathrm{C}_{1}\right), 144.2\left(\mathrm{~d},{ }^{3} J_{\mathrm{CP}}=6.5, \mathrm{C}=\right), 163.1\left(\mathrm{~d},{ }^{1} J_{\mathrm{CF}}=248.0, \mathrm{C}_{3}\right) ;[\mathrm{M}+\mathrm{H}]^{+}$found $=408.1269, \mathrm{C}_{22} \mathrm{H}_{20} \mathrm{~N}_{3} \mathrm{O}_{2} \mathrm{FP}$ requires 408.1277 .

[1-(4-Fluorobenzyl)-1H-1,2,3-triazol-4-yl]methyl diphenylphosphinate (13e): Yield: 83\% (0.34 g), pale yellow crystals; Mp: $95-97{ }^{\circ} \mathrm{C} ;{ }^{31} \mathrm{P} \mathrm{NMR}\left(\mathrm{CDCl}_{3}\right) \delta 33.5 ;{ }^{1} \mathrm{H}$ NMR $\left(\mathrm{CDCl}_{3}\right) \delta 5.18\left(\mathrm{~d},{ }^{3} J_{\mathrm{HP}}=9.2,2 \mathrm{H}\right.$, $\left.\mathrm{CH}_{2} \mathrm{O}\right), 5.43\left(\mathrm{~s}, 2 \mathrm{H}, \mathrm{CH}_{2} \mathrm{Ph}\right), 7.04\left(\mathrm{t}, J_{\mathrm{HF}}={ }^{3} J_{\mathrm{HH}}=8.6,2 \mathrm{H}, \mathrm{C}_{2} \mathrm{H}\right), 7.23\left(\mathrm{dd},{ }^{3} J_{\mathrm{HF}}=8.4,{ }^{3} J_{\mathrm{HH}}=5.1,2 \mathrm{H}\right.$, $\left.\mathrm{C}_{3} \mathrm{H}\right), 7.33-7.54\left(\mathrm{~m}, 6 \mathrm{H}, \mathrm{C}_{3}, \mathrm{H}, \mathrm{C}_{4}, \mathrm{H}\right), 7.55(\mathrm{~s}, 1 \mathrm{H}, \mathrm{CH}), 7.79\left(\mathrm{dd},{ }^{3} J_{\mathrm{HP}}=12.4,{ }^{3} J_{\mathrm{HH}}=7.4,4 \mathrm{H}, \mathrm{C}_{2}, \mathrm{H}\right)$; ${ }^{13} \mathrm{C}$ NMR $\left(\mathrm{CDCl}_{3}\right) \delta 53.5\left(\mathrm{CH}_{2} \mathrm{Ph}\right), 58.2\left(\mathrm{~d},{ }^{2} J_{\mathrm{CP}}=5.3, \mathrm{CH}_{2} \mathrm{O}\right), 116.2\left(\mathrm{~d},{ }^{2} J_{\mathrm{CF}}=21.9, \mathrm{C}_{3}\right), 123.8(\mathrm{CH}=)$, $128.7\left(\mathrm{~d},{ }^{3} J_{\mathrm{CP}}=13.3, \mathrm{C}_{3^{\prime}}\right), 130.1\left(\mathrm{~d},{ }^{3} J_{\mathrm{CF}}=8.4, \mathrm{C}_{2}\right), 130.4\left(\mathrm{~d}, J_{\mathrm{CF}}=3.3, \mathrm{C}_{1}\right), 131.2\left(\mathrm{~d},{ }^{1} J_{\mathrm{CP}}=136.7, \mathrm{C}_{1^{\prime}}\right)$, $131.8\left(\mathrm{~d},{ }^{2} J_{\mathrm{CP}}=10.3, \mathrm{C}_{2^{\prime}}\right), 132.5\left(\mathrm{~d}, J_{\mathrm{CP}}=2.9, \mathrm{C}_{4^{\prime}}\right), 144.2\left(\mathrm{~d},{ }^{3} J_{\mathrm{CP}}=6.2, \mathrm{C}=\right), 163.0\left(\mathrm{~d},{ }^{1} J_{\mathrm{CF}}=248.3, \mathrm{C}_{4}\right)$; $[\mathrm{M}+\mathrm{H}]^{+}$found $=408.1268, \mathrm{C}_{22} \mathrm{H}_{20} \mathrm{~N}_{3} \mathrm{O}_{2} \mathrm{FP}$ requires 408.1277.

[1-(4-Trifluoromethyl)benzyl)-1H-1,2,3-triazol-4-yl]methyl diphenylphosphinate (13f): Yield: $88 \%(0.40$ g), white crystals; Mp: $124-125{ }^{\circ} \mathrm{C} ;{ }^{31} \mathrm{P}$ NMR $\left(\mathrm{CDCl}_{3}\right) \delta 33.6 ;{ }^{1} \mathrm{H}$ NMR $\left(\mathrm{CDCl}_{3}\right) \delta 5.20\left(\mathrm{~d},{ }^{3} J_{\mathrm{HP}}=9.5\right.$, $\left.2 \mathrm{H}, \mathrm{CH}_{2} \mathrm{O}\right), 5.43\left(\mathrm{~s}, 2 \mathrm{H}, \mathrm{CH}_{2} \mathrm{Ph}\right), 7.33\left(\mathrm{~d},{ }^{3} \mathrm{~J}_{\mathrm{HH}}=8.0,2 \mathrm{H}, \mathrm{C}_{2} \mathrm{H}\right), 7.39-7.46\left(\mathrm{~m}, 4 \mathrm{H}, \mathrm{C}_{3}, \mathrm{H}\right), 7.48-7.53$ $\left(\mathrm{m}, 2 \mathrm{H}, \mathrm{C}_{4^{\prime}} \mathrm{H}\right), 7.61\left(\mathrm{~d},{ }^{3} J_{\mathrm{HH}}=8.0,2 \mathrm{H}, \mathrm{C}_{3} \mathrm{H}\right), 7.63(\mathrm{~s}, 1 \mathrm{H}, \mathrm{CH}), 7.79\left(\mathrm{dd},{ }^{3} J_{\mathrm{HP}}=12.4,{ }^{3} J_{\mathrm{HH}}=6.9,4 \mathrm{H}\right.$, $\left.\mathrm{C}_{2}, \mathrm{H}\right) ;{ }^{13} \mathrm{C}$ NMR $\left(\mathrm{CDCl}_{3}\right) \delta 53.6\left(\mathrm{CH}_{2} \mathrm{Ph}\right), 58.2\left(\mathrm{~d}^{2}{ }^{2} J_{\mathrm{CP}}=5.4, \mathrm{CH}_{2} \mathrm{O}\right), 122.8(\mathrm{CH}=), 123.9\left(\mathrm{q},{ }^{1} J_{\mathrm{CF}}=272.1\right.$, $\left.\mathrm{CF}_{3}\right), 126.2\left(\mathrm{q}^{3} \mathrm{~J}_{\mathrm{CF}}=3.7, \mathrm{C}_{3}\right), 128.4\left(\mathrm{C}_{2}\right), 128.7\left(\mathrm{~d},{ }^{3} J_{\mathrm{CP}}=13.3, \mathrm{C}_{3^{\prime}}\right), 131.1\left(\mathrm{q},{ }^{2} J_{\mathrm{CF}}=32.9, \mathrm{C}_{4}\right), 131.2(\mathrm{~d}$, $\left.{ }^{1} J_{\mathrm{CP}}=136.7, \mathrm{C}_{1^{\prime}}\right), 131.7\left(\mathrm{~d},{ }^{2} J_{\mathrm{CP}}=10.3, \mathrm{C}_{2^{\prime}}\right), 132.5\left(\mathrm{~d}, J_{\mathrm{CP}}=2.9, \mathrm{C}_{4^{\prime}}\right), 138.5\left(\mathrm{C}_{1}\right), 144.4\left(\mathrm{~d},{ }^{3} J_{\mathrm{CP}}=6.0, \mathrm{C}=\right)$; $[\mathrm{M}+\mathrm{H}]^{+}$found $=458.1242, \mathrm{C}_{23} \mathrm{H}_{20} \mathrm{~N}_{3} \mathrm{O}_{2} \mathrm{~F}_{3} \mathrm{P}$ requires 458.1245 .

(1-Octyl-1H-1,2,3-triazol-4-yl)methyl diphenylphosphinate (13g): Yield: 89\% (0.37 g), yellow oil; ${ }^{31} \mathrm{P}$ NMR $\left(\mathrm{CDCl}_{3}\right) \delta 33.2 ;{ }^{1} \mathrm{H}$ NMR $\left(\mathrm{CDCl}_{3}\right) \delta 0.87\left(\mathrm{t},{ }^{3} J_{\mathrm{HH}}=6.5,3 \mathrm{H}, \mathrm{CH}_{3}\right), 1.10-1.41$ $\left(\mathrm{m}, 10 \mathrm{H}, \mathrm{CH}_{2} \mathrm{CH}_{2}\left(\mathrm{CH}_{2}\right)_{5} \mathrm{CH}_{3}\right), 1.72-1.97\left(\mathrm{~m}, 2 \mathrm{H}, \mathrm{CH}_{2} \mathrm{CH}_{2}\left(\mathrm{CH}_{2}\right)_{5} \mathrm{CH}_{3}\right), 4.28\left(\mathrm{t}, 2 \mathrm{H},{ }^{3} J_{\mathrm{HH}}=7.3\right.$, $\left.\mathrm{CH}_{2} \mathrm{CH}_{2}\left(\mathrm{CH}_{2}\right)_{5} \mathrm{CH}_{3}\right), 5.21\left(\mathrm{~d},{ }^{3} J_{\mathrm{HP}}=9.1,2 \mathrm{H}, \mathrm{CH}_{2} \mathrm{O}\right), 7.34-7.57\left(\mathrm{~m}, 6 \mathrm{H}, \mathrm{C}_{3} \mathrm{H}, \mathrm{C}_{4} \mathrm{H}\right), 7.60(\mathrm{~s}, 1 \mathrm{H}$, $\mathrm{CH}), 7.82\left(\mathrm{dd},{ }^{3} J_{\mathrm{HP}}=12.4,{ }^{3} J_{\mathrm{HH}}=7.3,4 \mathrm{H}, \mathrm{C} 2 \mathrm{H}\right) ;{ }^{13} \mathrm{C} \mathrm{NMR}\left(\mathrm{CDCl}_{3}\right) \delta 14.2\left(\mathrm{CH}_{3}\right), 22.7\left(\mathrm{CH}_{2} \mathrm{CH}_{3}\right)$, $26.6\left(\mathrm{CH}_{2} \mathrm{CH}_{2} \mathrm{CH}_{3}\right), 29.0\left(\mathrm{CH}_{2}\left(\mathrm{CH}_{2}\right)_{2} \mathrm{CH}_{3}\right), 29.1\left(\mathrm{CH}_{2}\left(\mathrm{CH}_{2}\right)_{3} \mathrm{CH}_{3}\right), 30.3\left(\mathrm{CH}_{2}\left(\mathrm{CH}_{2}\right)_{4} \mathrm{CH}_{3}\right), 31.8$ $\left(\mathrm{CH}_{2}\left(\mathrm{CH}_{2}\right)_{5} \mathrm{CH}_{3}\right), 50.5\left(\mathrm{CH}_{2}\left(\mathrm{CH}_{2}\right)_{6} \mathrm{CH}_{3}\right), 58.3\left(\mathrm{~d},{ }^{2} J_{\mathrm{CP}}=5.3, \mathrm{CH}_{2} \mathrm{O}\right), 123.7(\mathrm{CH}=), 128.7\left(\mathrm{~d},{ }^{3} J_{\mathrm{CP}}=13.2\right.$, $\left.\mathrm{C}_{3}\right), 131.4\left(\mathrm{~d},{ }^{1} J_{\mathrm{CP}}=136.6, \mathrm{C}_{1}\right), 131.8\left(\mathrm{~d},{ }^{2} J_{\mathrm{CP}}=10.3, \mathrm{C}_{2}\right), 132.4\left(\mathrm{~d}, J_{\mathrm{CP}}=2.8, \mathrm{C}_{4}\right), 143.6\left(\mathrm{~d},{ }^{3} J_{\mathrm{CP}}=6.9\right.$, $\mathrm{C}=) ;[\mathrm{M}+\mathrm{H}]^{+}$found $=412.2154, \mathrm{C}_{23} \mathrm{H}_{31} \mathrm{~N}_{3} \mathrm{O}_{2} \mathrm{P}$ requires 412.2153 . 
(1-Iso-octyl-1H-1,2,3-triazol-4-yl)methyl diphenylphosphinate (13h): Yield: 77\% (0.49 g), yellow oil; ${ }^{31} \mathrm{P}$ $\operatorname{NMR}\left(\mathrm{CDCl}_{3}\right) \delta 33.3 ;{ }^{1} \mathrm{H}$ NMR $\left(\mathrm{CDCl}_{3}\right) \delta 0.89\left(\mathrm{t}^{3}{ }^{3} \mathrm{HH}=6.7,6 \mathrm{H}, \mathrm{CH}_{3}\right), 1.02-1.41\left(\mathrm{~m}, 8 \mathrm{H}, \mathrm{CH}\left(\mathrm{CH}_{2}\right)_{3} \mathrm{CH}_{3}\right.$, $\left.\mathrm{CHCH}_{2} \mathrm{CH}_{3}\right), 1.74-1.97\left(\mathrm{~m}, 1 \mathrm{H}, \mathrm{NCH}_{2} \mathrm{CH}\right), 4.21\left(\mathrm{~d}, 2 \mathrm{H},{ }^{3} J_{\mathrm{HH}}=6.9, \mathrm{NCH}_{2} \mathrm{CH}\right), 5.22\left(\mathrm{~d},{ }^{3} J_{\mathrm{HP}}=9.0,2 \mathrm{H}\right.$, $\left.\mathrm{CH}_{2} \mathrm{O}\right), 7.34-7.58\left(\mathrm{~m}, 6 \mathrm{H}, \mathrm{C}_{3} \mathrm{H}, \mathrm{C}_{4} \mathrm{H}\right), 7.59(\mathrm{~s}, 1 \mathrm{H}, \mathrm{CH}), 7.82\left(\mathrm{dd},{ }^{3} J_{\mathrm{HP}}=12.5,{ }^{3} J_{\mathrm{HH}}=7.4,4 \mathrm{H}, \mathrm{C}_{2} \mathrm{H}\right)$; ${ }^{13} \mathrm{C} \mathrm{NMR}\left(\mathrm{CDCl}_{3}\right) \delta 10.5\left(\mathrm{NCH}_{2} \mathrm{CHCH}_{2} \mathrm{CH}_{3}\right), 14.0\left(\mathrm{CH}_{3}\right), 22.9\left(\mathrm{CH}_{2} \mathrm{CH}_{3}\right), 23.7\left(\mathrm{NCH}_{2} \mathrm{CHCH}_{2} \mathrm{CH}_{3}\right)$, $28.5\left(\mathrm{CH}_{2} \mathrm{CH}_{2} \mathrm{CH}_{3}\right), 30.4\left(\mathrm{CH}_{2}\left(\mathrm{CH}_{2}\right)_{2} \mathrm{CH}_{3}\right), 40.3\left(\mathrm{NCH}_{2} \mathrm{CHCH}_{2} \mathrm{CH}_{3}\right), 53.6\left(\mathrm{NCH}_{2} \mathrm{CHCH}_{2} \mathrm{CH}_{3}\right), 58.2$ $\left(\mathrm{d},{ }^{2} J_{\mathrm{CP}}=5.3, \mathrm{CH}_{2} \mathrm{O}\right), 124.2(\mathrm{CH}=), 128.6\left(\mathrm{~d},{ }^{3} J_{\mathrm{CP}}=13.3, \mathrm{C}_{3}\right), 131.3\left(\mathrm{~d},{ }^{1} J_{\mathrm{CP}}=136.7, \mathrm{C}_{1}\right), 131.7(\mathrm{~d}$, $\left.{ }^{2} J_{\mathrm{CP}}=10.3, \mathrm{C}_{2}\right), 132.4\left(\mathrm{~d}, J_{\mathrm{CP}}=2.9, \mathrm{C}_{4}\right), 143.4\left(\mathrm{~d},{ }^{3} J_{\mathrm{CP}}=6.2, \mathrm{C}=\right) ;[\mathrm{M}+\mathrm{H}]^{+}$found $=412.2154, \mathrm{C}_{23} \mathrm{H}_{31} \mathrm{~N}_{3} \mathrm{O}_{2} \mathrm{P}$ requires 412.2153 .

(1-Cyclohexyl-1H-1,2,3-triazol-4-yl)methyl diphenylphosphinate (13i): Yield: 63\% (0.24 g), white crystals; Mp: $122-124{ }^{\circ} \mathrm{C} ;{ }^{31} \mathrm{P}$ NMR $\left(\mathrm{CDCl}_{3}\right) \delta 33.2 ;{ }^{1} \mathrm{H}$ NMR $\left(\mathrm{CDCl}_{3}\right) \delta 1.16-1.52\left(\mathrm{~m}, 4 \mathrm{H}, \mathrm{C}_{3} \mathrm{H}_{\mathrm{ax}}\right.$ $\left.\mathrm{C}_{4} \mathrm{H}_{\mathrm{ax}}, \mathrm{C}_{4} \mathrm{H}_{\mathrm{eq}}\right), 1.58-1.80\left(\mathrm{~m}, 2 \mathrm{H}, \mathrm{C}_{3} \mathrm{H}_{\mathrm{eq}}\right), 1.81-1.95\left(\mathrm{~m}, 2 \mathrm{H}, \mathrm{C}_{2} \mathrm{H}_{\mathrm{ax}}\right), 2.01-2.22\left(\mathrm{~m}, 2 \mathrm{H}, \mathrm{C}_{2} \mathrm{H}_{\mathrm{eq}}\right), 4.29-4.45$ $\left(\mathrm{m}, 1 \mathrm{H}, \mathrm{C}_{1} \mathrm{H}\right), 5.21\left(\mathrm{~d},{ }^{3} J_{\mathrm{HP}}=9.0,2 \mathrm{H}, \mathrm{CH}_{2} \mathrm{O}\right), 7.32-7.56\left(\mathrm{~m}, 6 \mathrm{H}, \mathrm{C}_{3^{\prime}} \mathrm{H}, \mathrm{C}_{4^{\prime}} \mathrm{H}\right), 7.59(\mathrm{~s}, 1 \mathrm{H}, \mathrm{CH}), 7.82$ $\left(\mathrm{dd},{ }^{3} J_{\mathrm{HP}}=12.4,{ }^{3} J_{\mathrm{HH}}=7.3,4 \mathrm{H}, \mathrm{C}_{2}, \mathrm{H}\right) ;{ }^{13} \mathrm{C} \mathrm{NMR}\left(\mathrm{CDCl}_{3}\right) \delta 25.2\left(\mathrm{C}_{4}\right), 25.3\left(\mathrm{C}_{3}\right), 33.6\left(\mathrm{C}_{2}\right), 58.4(\mathrm{~d}$, $\left.{ }^{2} J_{\mathrm{CP}}=5.3, \mathrm{CH}_{2} \mathrm{O}\right), 60.3\left(\mathrm{C}_{1}\right) 121.6(\mathrm{CH}=), 128.7\left(\mathrm{~d},{ }^{3} J_{\mathrm{CP}}=13.2, \mathrm{C}_{3^{\prime}}\right), 131.4\left(\mathrm{~d},{ }^{1} J_{\mathrm{CP}}=136.9, \mathrm{C}_{1^{\prime}}\right)$, $131.8\left(\mathrm{~d},{ }^{2} J_{\mathrm{CP}}=10.3, \mathrm{C}_{2^{\prime}}\right), 132.4\left(\mathrm{~d}, J_{\mathrm{CP}}=2.8, \mathrm{C}_{4^{\prime}}\right), 143.1\left(\mathrm{~d},{ }^{3} J_{\mathrm{CP}}=6.5, \mathrm{C}=\right) ;[\mathrm{M}+\mathrm{H}]^{+}$found $=382.1677$, $\mathrm{C}_{21} \mathrm{H}_{25} \mathrm{~N}_{3} \mathrm{O}_{2}$ P requires 382.1684 .

(1-Phenyl-1H-1,2,3-triazol-4-yl)methyl diphenylphosphinate (13j): Yield: $82 \%(0.31 \mathrm{~g})$, white crystals; Mp: $121-122{ }^{\circ} \mathrm{C} ;{ }^{31} \mathrm{P}$ NMR $\left(\mathrm{CDCl}_{3}\right) \delta 33.5 ;{ }^{1} \mathrm{H}$ NMR $\left(\mathrm{CDCl}_{3}\right) \delta 5.30\left(\mathrm{~d},{ }^{3} \mathrm{~J}_{\mathrm{HP}}=9.1,2 \mathrm{H}, \mathrm{CH}_{2} \mathrm{O}\right), 7.34-7.57$ $\left(\mathrm{m}, 9 \mathrm{H}, \mathrm{C}_{3} \mathrm{H}, \mathrm{C}_{4} \mathrm{H}, \mathrm{C}_{3^{\prime}} \mathrm{H}, \mathrm{C}_{4}, \mathrm{H}\right), 7.68\left(\mathrm{~d},{ }^{3} J_{\mathrm{HH}}=7.7,2 \mathrm{H}, \mathrm{C}_{2} \mathrm{H}\right), 7.85\left(\mathrm{dd},{ }^{3} J_{\mathrm{HP}}=12.4,{ }^{3} J_{\mathrm{HH}}=7.4,4 \mathrm{H}\right.$, $\left.\mathrm{C}_{2}, \mathrm{H}\right), 8.05(\mathrm{~s}, 1 \mathrm{H}, \mathrm{CH}) ;{ }^{13} \mathrm{C} \mathrm{NMR}\left(\mathrm{CDCl}_{3}\right) \delta 58.2\left(\mathrm{~d},{ }^{2} J_{\mathrm{CP}}=5.3, \mathrm{CH}_{2} \mathrm{O}\right), 120.7\left(\mathrm{C}_{2}\right), 122.2(\mathrm{CH}=), 128.8$ $\left(\mathrm{d},{ }^{3} J_{\mathrm{CP}}=13.2, \mathrm{C}_{3^{\prime}}\right), 129.0\left(\mathrm{C}_{4}\right), 129.9\left(\mathrm{C}_{3}\right), 131.2\left(\mathrm{~d},{ }^{1} J_{\mathrm{CP}}=136.6, \mathrm{C}_{1^{\prime}}\right), 131.8\left(\mathrm{~d},{ }^{2} J_{\mathrm{CP}}=10.4, \mathrm{C}_{2^{\prime}}\right)$, $132.5\left(\mathrm{~d}, J_{\mathrm{CP}}=2.9, \mathrm{C}_{4^{\prime}}\right), 137.0\left(\mathrm{C}_{1}\right), 144.4\left(\mathrm{~d},{ }^{3} J_{\mathrm{CP}}=6.0, \mathrm{C}=\right) ;[\mathrm{M}+\mathrm{H}]^{+}$found $=376.1210, \mathrm{C}_{21} \mathrm{H}_{19} \mathrm{~N}_{3} \mathrm{O}_{2} \mathrm{P}$ requires 376.1214 .

(1-Benzyl-1H-1,2,3-triazol-4-yl)methyl diethyl phosphate (14a): Yield: 75\% (0.24 g), pale yellow oil; ${ }^{31} \mathrm{P} \mathrm{NMR}\left(\mathrm{CDCl}_{3}\right) \delta-1.0 ;{ }^{1} \mathrm{H}$ NMR $\left(\mathrm{CDCl}_{3}\right) \delta 1.27\left(\mathrm{t},{ }^{3} \mathrm{~J}_{\mathrm{HH}}=6.9,6 \mathrm{H}, \mathrm{OCH}_{2} \mathrm{CH}_{3}\right), 4.01-4.09(\mathrm{~m}, 4 \mathrm{H}$, $\left.\mathrm{OCH}_{2} \mathrm{CH}_{3}\right), 5.16\left(\mathrm{~d},{ }^{3} J_{\mathrm{HP}}=9.4,2 \mathrm{H}, \mathrm{CH}_{2} \mathrm{O}\right), 5.53\left(\mathrm{~s}, 2 \mathrm{H}, \mathrm{CH}_{2} \mathrm{Ph}\right), 7.25-7.30\left(\mathrm{~m}, 2 \mathrm{H}, \mathrm{C}_{2} \mathrm{H}\right), 7.32-7.40(\mathrm{~m}$, $\left.3 \mathrm{H}, \mathrm{C}_{3} \mathrm{H}, \mathrm{C}_{4} \mathrm{H}\right), 7.62(\mathrm{~s}, 1 \mathrm{H}, \mathrm{CH}) ;{ }^{13} \mathrm{C} \mathrm{NMR}\left(\mathrm{CDCl}_{3}\right) \delta 16.1\left(\mathrm{~d},{ }^{3} J_{\mathrm{CP}}=6.3, \mathrm{OCH}_{2} \mathrm{CH}_{3}\right), 54.4\left(\mathrm{CH}_{2} \mathrm{Ph}\right)$, $60.6\left(\mathrm{~d},{ }^{2} J_{\mathrm{CP}}=6.2, \mathrm{CH}_{2} \mathrm{O}\right), 64.1\left(\mathrm{~d},{ }^{2} J_{\mathrm{CP}}=6.4, \mathrm{OCH}_{2} \mathrm{CH}_{3}\right), 123.5(\mathrm{CH}=), 128.2\left(\mathrm{C}_{2}\right), 129.0\left(\mathrm{C}_{4}\right), 129.2$ $\left(\mathrm{C}_{3}\right), 134.5\left(\mathrm{C}_{1}\right), 143.8\left(\mathrm{~d},{ }^{3} J_{\mathrm{CP}}=8.2, \mathrm{C}=\right) ;[\mathrm{M}+\mathrm{H}]^{+}$found $=326.1259, \mathrm{C}_{14} \mathrm{H}_{21} \mathrm{~N}_{3} \mathrm{O}_{4} \mathrm{P}$ requires 326.1269.

[1-(4-Methylbenzyl)-1H-1,2,3-triazol-4-yl]methyl diethyl phosphate (14b): Yield: 69\% (0.23 g), pale yellow oil; ${ }^{31} \mathrm{P}$ NMR $\left(\mathrm{CDCl}_{3}\right) \delta-1.1 ;{ }^{1} \mathrm{H}$ NMR $\left(\mathrm{CDCl}_{3}\right) \delta 1.17\left(\mathrm{t},{ }^{3} \mathrm{~J}_{\mathrm{HH}}=6.9,6 \mathrm{H}, \mathrm{OCH}_{2} \mathrm{CH}_{3}\right), 2.27(\mathrm{~s}$, $\left.3 \mathrm{H}, \mathrm{CH}_{3} \mathrm{Ph}\right), 3.87-4.05\left(\mathrm{~m}, 4 \mathrm{H}, \mathrm{OCH}_{2} \mathrm{CH}_{3}\right), 5.07\left(\mathrm{~d},{ }^{3} J_{\mathrm{HP}}=9.2,2 \mathrm{H}, \mathrm{CH}_{2} \mathrm{O}\right), 5.40\left(\mathrm{~s}, 2 \mathrm{H}, \mathrm{CH}_{2} \mathrm{Ph}\right), 7.10(\mathrm{~s}$, $\left.4 \mathrm{H}, \mathrm{C}_{2} \mathrm{H}, \mathrm{C}_{3} \mathrm{H}\right), 7.52(\mathrm{~s}, 1 \mathrm{H}, \mathrm{CH}) ;{ }^{13} \mathrm{C} \mathrm{NMR}\left(\mathrm{CDCl}_{3}\right) \delta 16.1\left(\mathrm{~d},{ }^{3} J_{\mathrm{CP}}=6.8, \mathrm{OCH}_{2} \mathrm{CH}_{3}\right), 21.2\left(\mathrm{CH}_{3} \mathrm{Ph}\right)$, $54.1\left(\mathrm{CH}_{2} \mathrm{Ph}\right), 60.6\left(\mathrm{~d},{ }^{2} J_{\mathrm{CP}}=5.2, \mathrm{CH}_{2} \mathrm{O}\right), 64.0\left(\mathrm{~d},{ }^{2} J_{\mathrm{CP}}=5.9, \mathrm{OCH}_{2} \mathrm{CH}_{3}\right), 123.3(\mathrm{CH}=), 128.3\left(\mathrm{C}_{2}\right)$, $129.9\left(\mathrm{C}_{3}\right), 131.5\left(\mathrm{C}_{1}\right), 138.9\left(\mathrm{C}_{4}\right), 143.7\left(\mathrm{~d},{ }^{3} J_{\mathrm{CP}}=6.9, \mathrm{C}=\right)$; $[\mathrm{M}+\mathrm{H}]^{+}$found $=340.1414, \mathrm{C}_{15} \mathrm{H}_{23} \mathrm{~N}_{3} \mathrm{O}_{4} \mathrm{P}$ requires 340.1426 .

[1-(2-Fluorobenzyl)-1H-1,2,3-triazol-4-yl]methyl diethyl phosphate (14c): Yield: 54\% (0.19 g), pale yellow oil; ${ }^{31} \mathrm{P}$ NMR $\left(\mathrm{CDCl}_{3}\right) \delta-1.0 ;{ }^{1} \mathrm{H}$ NMR $\left(\mathrm{CDCl}_{3}\right) \delta 1.28\left(\mathrm{t},{ }^{3} \mathrm{JHH}_{\mathrm{HH}}=7.1,6 \mathrm{H}, \mathrm{OCH}_{2} \mathrm{CH}_{3}\right), 3.97-4.17$ $\left(\mathrm{m}, 4 \mathrm{H}, \mathrm{OCH}_{2} \mathrm{CH}_{3}\right), 5.16\left(\mathrm{~d},{ }^{3} \mathrm{~J}_{\mathrm{HP}}=9.3,2 \mathrm{H}, \mathrm{CH}_{2} \mathrm{O}\right), 5.59\left(\mathrm{~s}, 2 \mathrm{H}, \mathrm{CH}_{2} \mathrm{Ph}\right), 7.07-7.20\left(\mathrm{~m}, 2 \mathrm{H}, \mathrm{C}_{3} \mathrm{H}, \mathrm{C} 5 \mathrm{H}\right)$, 7.24-7.31 (m, 1H, $\left.\mathrm{C}_{6} \mathrm{H}\right), 7.32-7.41\left(\mathrm{~m}, 1 \mathrm{H}, \mathrm{C}_{4} \mathrm{H}\right), 7.70(\mathrm{~s}, 1 \mathrm{H}, \mathrm{CH}) ;{ }^{13} \mathrm{C} \mathrm{NMR}\left(\mathrm{CDCl}_{3}\right) \delta 16.2\left(\mathrm{~d}^{3}{ }^{3} \mathrm{JP}_{\mathrm{CP}}=6.8\right.$, $\left.\mathrm{OCH}_{2} \mathrm{CH}_{3}\right), 47.9\left(\mathrm{~d},{ }^{3} J_{\mathrm{CF}}=4.3, \mathrm{CH}_{2} \mathrm{Ph}\right), 60.6\left(\mathrm{~d},{ }^{2} J_{\mathrm{CP}}=5.3, \mathrm{CH}_{2} \mathrm{O}\right), 64.1\left(\mathrm{~d},{ }^{2} J_{\mathrm{CP}}=5.9, \mathrm{OCH}_{2} \mathrm{CH}_{3}\right), 116.0$ $\left(\mathrm{d},{ }^{2} J_{\mathrm{CF}}=21.1, \mathrm{C}_{3}\right), 121.8\left(\mathrm{~d},{ }^{2} J_{\mathrm{CF}}=14.6, \mathrm{C}_{1}\right), 123.6(\mathrm{CH}=), 125.0\left(\mathrm{~d}, J_{\mathrm{CF}}=3.7, \mathrm{C}_{5}\right), 130.8\left(\mathrm{~d},{ }^{3} J_{\mathrm{CF}}=3.2, \mathrm{C}_{4}\right)$, $131.2\left(\mathrm{~d},{ }^{3} J_{\mathrm{CF}}=8.3, \mathrm{C}_{6}\right), 143.9\left(\mathrm{~d},{ }^{3} J_{\mathrm{CP}}=7.0, \mathrm{C}=\right), 160.7\left(\mathrm{~d},{ }^{1} J_{\mathrm{CF}}=248.0, \mathrm{C}_{2}\right) ;[\mathrm{M}+\mathrm{H}]^{+}$found $=344.1164$, $\mathrm{C}_{14} \mathrm{H}_{20} \mathrm{~N}_{3} \mathrm{O}_{4}$ FP requires 344.1175 .

[1-(3-Fluorobenzyl)-1H-1,2,3-triazol-4-yl]methyl diethyl phosphate (14d): Yield: 56\% (0.20 g), pale yellow oil; ${ }^{31} \mathrm{P}$ NMR $\left(\mathrm{CDCl}_{3}\right) \delta-0.9 ;{ }^{1} \mathrm{H}$ NMR $\left(\mathrm{CDCl}_{3}\right) \delta 1.28\left(\mathrm{t},{ }^{3} \mathrm{~J}_{\mathrm{HH}}=7.1,6 \mathrm{H}, \mathrm{OCH}_{2} \mathrm{CH}_{3}\right), 3.95-4.15$ $\left(\mathrm{m}, 4 \mathrm{H}, \mathrm{OCH}_{2} \mathrm{CH}_{3}\right), 5.17\left(\mathrm{~d},{ }^{3} J_{\mathrm{HP}}=9.5,2 \mathrm{H}, \mathrm{CH}_{2} \mathrm{O}\right), 5.53\left(\mathrm{~s}, 2 \mathrm{H}, \mathrm{CH}_{2} \mathrm{Ph}\right), 6.89-7.00(\mathrm{~m}, 1 \mathrm{H}, \mathrm{C} 4 \mathrm{H})$, 7.00-7.12 (m, 2H, $\left.\mathrm{C}_{2} \mathrm{H}, \mathrm{C}_{6} \mathrm{H}\right), 7.26-7.42\left(\mathrm{~m}, 1 \mathrm{H}, \mathrm{C}_{5} \mathrm{H}\right), 7.66(\mathrm{~s}, 1 \mathrm{H}, \mathrm{CH}) ;{ }^{13} \mathrm{C}$ NMR $\left(\mathrm{CDCl}_{3}\right) \delta 16.2(\mathrm{~d}$, $\left.{ }^{3} J_{\mathrm{CP}}=6.8, \mathrm{OCH}_{2} \mathrm{CH}_{3}\right), 53.7\left(\mathrm{~d}, J_{\mathrm{CF}}=1.9, \mathrm{CH}_{2} \mathrm{Ph}\right), 60.6\left(\mathrm{~d},{ }^{2} J_{\mathrm{CP}}=5.2, \mathrm{CH}_{2} \mathrm{O}\right), 64.1\left(\mathrm{~d},{ }^{2} J_{\mathrm{CP}}=6.0\right.$, 
$\left.\mathrm{OCH}_{2} \mathrm{CH}_{3}\right), 115.2\left(\mathrm{~d},{ }^{2} J_{\mathrm{CF}}=22.3, \mathrm{C}_{4}\right), 116.1\left(\mathrm{~d},{ }^{2} J_{\mathrm{CF}}=21.0, \mathrm{C}_{2}\right), 123.6(\mathrm{CH}=), 123.7\left(\mathrm{~d}, J_{\mathrm{CF}}=3.1, \mathrm{C}_{6}\right)$, $131.0\left(\mathrm{~d},{ }^{3} J_{\mathrm{CF}}=8.3, \mathrm{C}_{5}\right), 136.9\left(\mathrm{~d},{ }^{3} J_{\mathrm{CF}}=7.4, \mathrm{C}_{1}\right), 144.2\left(\mathrm{~d},{ }^{3} J_{\mathrm{CP}}=6.8, \mathrm{C}=\right), 163.2\left(\mathrm{~d},{ }^{1} J_{\mathrm{CF}}=248.1, \mathrm{C}_{3}\right)$; $[\mathrm{M}+\mathrm{H}]^{+}$found $=344.1164, \mathrm{C}_{14} \mathrm{H}_{20} \mathrm{~N}_{3} \mathrm{O}_{4} \mathrm{FP}$ requires 344.1175.

[1-(4-Fluorobenzyl)-1H-1,2,3-triazol-4-yl])methyl diethyl phosphate (14e): Yield: 61\% (0.22 g), pale yellow oil; ${ }^{31} \mathrm{P}$ NMR $\left(\mathrm{CDCl}_{3}\right) \delta-1.0 ;{ }^{1} \mathrm{H}$ NMR $\left(\mathrm{CDCl}_{3}\right) \delta 1.28\left(\mathrm{t},{ }^{3} \mathrm{~J}_{\mathrm{HH}}=7.1,6 \mathrm{H}, \mathrm{OCH}_{2} \mathrm{CH}_{3}\right), 3.88-4.15$ $\left(\mathrm{m}, 4 \mathrm{H}, \mathrm{OCH}_{2} \mathrm{CH}_{3}\right), 5.16\left(\mathrm{~d},{ }^{3} J_{\mathrm{HP}}=9.5,2 \mathrm{H}, \mathrm{CH}_{2} \mathrm{O}\right), 5.50\left(\mathrm{~s}, 2 \mathrm{H}, \mathrm{CH}_{2} \mathrm{Ph}\right), 7.06\left(\mathrm{t}, J_{\mathrm{HF}}={ }^{3} J_{\mathrm{HH}}=8.6\right.$, $\left.2 \mathrm{H}, \mathrm{C}_{2} \mathrm{H}\right), 7.28\left(\mathrm{dd},{ }^{3} J_{\mathrm{HF}}=8.0,{ }^{3} J_{\mathrm{HH}}=5.8,2 \mathrm{H}, \mathrm{C}_{3} \mathrm{H}\right), 7.63(\mathrm{~s}, 1 \mathrm{H}, \mathrm{CH}) ;{ }^{13} \mathrm{C} \mathrm{NMR}\left(\mathrm{CDCl}_{3}\right) \delta 16.2(\mathrm{~d}$, $\left.{ }^{3} J_{\mathrm{CP}}=6.8, \mathrm{OCH}_{2} \mathrm{CH}_{3}\right), 53.6\left(\mathrm{CH}_{2} \mathrm{Ph}\right), 60.6\left(\mathrm{~d},{ }^{2} J_{\mathrm{CP}}=5.2, \mathrm{CH}_{2} \mathrm{O}\right), 64.1\left(\mathrm{~d},{ }^{2} J_{\mathrm{CP}}=6.0, \mathrm{OCH}_{2} \mathrm{CH}_{3}\right), 116.3$ $\left(\mathrm{d},{ }^{2} J_{\mathrm{CF}}=21.8, \mathrm{C}_{3}\right), 123.4(\mathrm{CH}=), 130.2\left(\mathrm{~d},{ }^{3} J_{\mathrm{CF}}=8.4, \mathrm{C}_{2}\right), 130.4\left(\mathrm{~d}, J_{\mathrm{CF}}=3.3, \mathrm{C}_{1}\right), 144.0\left(\mathrm{~d},{ }^{3} J_{\mathrm{CP}}=6.9\right.$, $\mathrm{C}=), 163.1\left(\mathrm{~d},{ }^{1} J_{\mathrm{CF}}=248.3, \mathrm{C}_{4}\right) ;[\mathrm{M}+\mathrm{H}]^{+}$found $=344.1163, \mathrm{C}_{14} \mathrm{H}_{20} \mathrm{~N}_{3} \mathrm{O}_{4} \mathrm{FP}$ requires 344.1175.

[1-(4-Trifluoromethyl)benzyl)-1H-1,2,3-triazol-4-yl]methyl diethyl phosphate (14f): Yield: $68 \%(0.27$ g), pale yellow oil; ${ }^{31} \mathrm{P}$ NMR $\left(\mathrm{CDCl}_{3}\right) \delta-0.9 ;{ }^{1} \mathrm{H} \mathrm{NMR}\left(\mathrm{CDCl}_{3}\right) \delta 1.23\left(\mathrm{t},{ }^{3} \mathrm{~J}_{\mathrm{HH}}=7.1,6 \mathrm{H}, \mathrm{OCH}_{2} \mathrm{CH}_{3}\right)$, $3.92-4.12\left(\mathrm{~m}, 4 \mathrm{H}, \mathrm{OCH}_{2} \mathrm{CH}_{3}\right), 5.13\left(\mathrm{~d},{ }^{3} J_{\mathrm{HP}}=9.4,2 \mathrm{H}, \mathrm{CH}_{2} \mathrm{O}\right), 5.57\left(\mathrm{~s}, 2 \mathrm{H}, \mathrm{CH}_{2} \mathrm{Ph}\right), 7.36\left(\mathrm{~d},{ }^{3} J_{\mathrm{HH}}=8.0\right.$, $\left.2 \mathrm{H}, \mathrm{C}_{2} \mathrm{H}\right), 7.59\left(\mathrm{~d},{ }^{3} J_{\mathrm{HH}}=8.0,2 \mathrm{H}, \mathrm{C}_{3} \mathrm{H}\right), 7.68(\mathrm{~s}, 1 \mathrm{H}, \mathrm{CH}) ;{ }^{13} \mathrm{C} \mathrm{NMR}\left(\mathrm{CDCl}_{3}\right) \delta 16.1\left(\mathrm{~d},{ }^{3} J_{\mathrm{CP}}=6.7\right.$, $\left.\mathrm{OCH}_{2} \mathrm{CH}_{3}\right), 53.6\left(\mathrm{CH}_{2} \mathrm{Ph}\right), 60.5\left(\mathrm{~d},{ }^{2} J_{\mathrm{CP}}=5.2, \mathrm{CH}_{2} \mathrm{O}\right), 64.0\left(\mathrm{~d},{ }^{2} J_{\mathrm{CP}}=5.9, \mathrm{OCH}_{2} \mathrm{CH}_{3}\right), 123.7(\mathrm{CH}=), 123.8$ $\left(\mathrm{q},{ }^{1} J_{\mathrm{CF}}=272.4, \mathrm{CF}_{3}\right), 126.1\left(\mathrm{q},{ }^{3} J_{\mathrm{CF}}=3.8, \mathrm{C}_{3}\right), 128.4\left(\mathrm{C}_{2}\right), 131.1\left(\mathrm{q},{ }^{2} J_{\mathrm{CF}}=32.8, \mathrm{C}_{4}\right), 138.6\left(\mathrm{C}_{1}\right), 144.1(\mathrm{~d}$, $\left.{ }^{3} J_{\mathrm{CP}}=6.8, \mathrm{C}=\right) ;[\mathrm{M}+\mathrm{H}]^{+}$found $=394.1157, \mathrm{C}_{15} \mathrm{H}_{20} \mathrm{~N}_{3} \mathrm{O}_{4} \mathrm{~F}_{3}$ P requires 394.1143 .

(1-Octyl-1H-1,2,3-triazol-4-yl)methyl diethyl phosphate (14g): Yield: $60 \%(0.21 \mathrm{~g})$, pale yellow oil; ${ }^{31} \mathrm{P}$ NMR $\left(\mathrm{CDCl}_{3}\right) \delta-1.3 ;{ }^{1} \mathrm{H}$ NMR $\left(\mathrm{CDCl}_{3}\right) \delta 0.88\left(\mathrm{t},{ }^{3} J_{\mathrm{HH}}=6.7,3 \mathrm{H}, \mathrm{CH}_{3}\right), 1.16-1.43(\mathrm{~m}, 16 \mathrm{H}$, $\left.\mathrm{CH}_{2} \mathrm{CH}_{2}\left(\mathrm{CH}_{2}\right)_{5} \mathrm{CH}_{3}, \mathrm{OCH}_{2} \mathrm{CH}_{3}\right), 1.81-2.01\left(\mathrm{~m}, 2 \mathrm{H}, \mathrm{CH}_{2} \mathrm{CH}_{2}\left(\mathrm{CH}_{2}\right)_{5} \mathrm{CH}_{3}\right), 4.01-4.22\left(\mathrm{~m}, 4 \mathrm{H}, \mathrm{OCH}_{2} \mathrm{CH}_{3}\right)$, $4.35\left(\mathrm{t}, 2 \mathrm{H},{ }^{3} \mathrm{~J}_{\mathrm{HH}}=7.3, \mathrm{CH}_{2} \mathrm{CH}_{2}\left(\mathrm{CH}_{2}\right)_{5} \mathrm{CH}_{3}\right), 5.19\left(\mathrm{~d},{ }^{3} \mathrm{~J}_{\mathrm{HP}}=9.4,2 \mathrm{H}, \mathrm{CH}_{2} \mathrm{O}\right), 7.68(\mathrm{~s}, 1 \mathrm{H}, \mathrm{CH}) ;{ }^{13} \mathrm{C}$ NMR $\left(\mathrm{CDCl}_{3}\right) \delta 14.2\left(\mathrm{CH}_{3}\right), 16.2\left(\mathrm{~d},{ }^{3} J_{\mathrm{CP}}=6.8, \mathrm{OCH}_{2} \mathrm{CH}_{3}\right), 22.7\left(\mathrm{CH}_{2} \mathrm{CH}_{3}\right), 26.6\left(\mathrm{CH}_{2} \mathrm{CH}_{2} \mathrm{CH}_{3}\right)$, $29.1\left(\mathrm{CH}_{2}\left(\mathrm{CH}_{2}\right)_{2} \mathrm{CH}_{3}\right), 29.2\left(\mathrm{CH}_{2}\left(\mathrm{CH}_{2}\right)_{3} \mathrm{CH}_{3}\right), 30.4\left(\mathrm{CH}_{2}\left(\mathrm{CH}_{2}\right)_{4} \mathrm{CH}_{3}\right), 31.8\left(\mathrm{CH}_{2}\left(\mathrm{CH}_{2}\right)_{5} \mathrm{CH}_{3}\right), 50.6$ $\left(\mathrm{CH}_{2}\left(\mathrm{CH}_{2}\right)_{6} \mathrm{CH}_{3}\right), 60.7\left(\mathrm{~d},{ }^{2} J_{\mathrm{CP}}=5.3, \mathrm{CH}_{2} \mathrm{O}\right), 64.1\left(\mathrm{~d},{ }^{2} J_{\mathrm{CP}}=6.0, \mathrm{OCH}_{2} \mathrm{CH}_{3}\right), 123.4(\mathrm{CH}=), 143.4(\mathrm{~d}$, $\left.{ }^{3} J_{\mathrm{CP}}=6.7, \mathrm{C}=\right) ;[\mathrm{M}+\mathrm{H}]^{+}$found $=348.2043, \mathrm{C}_{15} \mathrm{H}_{31} \mathrm{~N}_{3} \mathrm{O}_{4} \mathrm{P}$ requires 348.2052.

(1-Iso-octyl-1H-1,2,3-triazol-4-yl)methyl diethyl phosphate (14h): Yield: $54 \%$ (0.19 g), pale yellow oil; ${ }^{31} \mathrm{P}$ NMR $\left(\mathrm{CDCl}_{3}\right) \delta-1.2 ;{ }^{1} \mathrm{H}$ NMR $\left(\mathrm{CDCl}_{3}\right) \delta 0.91\left(\mathrm{t},{ }^{3} \mathrm{~J}_{\mathrm{HH}}=7.4,6 \mathrm{H}, \mathrm{CH}_{3}\right), 1.12-1.42(\mathrm{~m}, 14 \mathrm{H}$, $\left.\mathrm{CH}\left(\mathrm{CH}_{2}\right)_{3} \mathrm{CH}_{3}, \mathrm{CHCH}_{2} \mathrm{CH}_{3}, \mathrm{OCH}_{2} \mathrm{CH}_{3}\right), 1.80-1.95\left(\mathrm{~m}, 1 \mathrm{H}, \mathrm{NCH}_{2} \mathrm{CH}\right), 3.98-4.15\left(\mathrm{~m}, 4 \mathrm{H}, \mathrm{OCH}_{2} \mathrm{CH}_{3}\right)$, $4.27\left(\mathrm{~d}, 2 \mathrm{H},{ }^{3} J_{\mathrm{HH}}=6.8, \mathrm{NCH}_{2} \mathrm{CH}\right), 5.19\left(\mathrm{~d},{ }^{3} J_{\mathrm{HP}}=9.4,2 \mathrm{H}, \mathrm{CH}_{2} \mathrm{O}\right), 7.66(\mathrm{~s}, 1 \mathrm{H}, \mathrm{CH}) ;{ }^{13} \mathrm{C} \mathrm{NMR}$ $\left(\mathrm{CDCl}_{3}\right) \delta 10.6\left(\mathrm{NCH}_{2} \mathrm{CHCH}_{2} \mathrm{CH}_{3}\right), 14.1\left(\mathrm{CH}_{3}\right), 16.2\left(\mathrm{~d},{ }^{3} J_{\mathrm{CP}}=6.7, \mathrm{OCH}_{2} \mathrm{CH}_{3}\right), 23.0\left(\mathrm{CH}_{2} \mathrm{CH}_{3}\right)$, $23.8\left(\mathrm{NCH}_{2} \mathrm{CHCH}_{2} \mathrm{CH}_{3}\right), 28.6\left(\mathrm{CH}_{2} \mathrm{CH}_{2} \mathrm{CH}_{3}\right), 30.5\left(\mathrm{CH}_{2}\left(\mathrm{CH}_{2}\right)_{2} \mathrm{CH}_{3}\right), 40.5\left(\mathrm{NCH}_{2} \mathrm{CHCH}_{2} \mathrm{CH}_{3}\right), 53.7$ $\left(\mathrm{NCH}_{2} \mathrm{CHCH}_{2} \mathrm{CH}_{3}\right), 60.7\left(\mathrm{~d},{ }^{2} J_{\mathrm{CP}}=5.3, \mathrm{CH}_{2} \mathrm{O}\right), 64.1\left(\mathrm{~d},{ }^{2} J_{\mathrm{CP}}=6.0, \mathrm{OCH}_{2} \mathrm{CH}_{3}\right), 124.0(\mathrm{CH}=), 143.3$ $\left(\mathrm{d},{ }^{3} J_{\mathrm{CP}}=6.6, \mathrm{C}=\right) ;[\mathrm{M}+\mathrm{H}]^{+}$found $=348.2043, \mathrm{C}_{15} \mathrm{H}_{31} \mathrm{~N}_{3} \mathrm{O}_{4}$ P requires 348.2052.

(1-Cyclohexyl-1H-1,2,3-triazol-4-yl)methyl diethyl phosphate (14i): Yield: 51\% (0.16 g), pale yellow oil; ${ }^{31} \mathrm{P}$ NMR $\left(\mathrm{CDCl}_{3}\right) \delta-1.3 ;{ }^{1} \mathrm{H}$ NMR $\left(\mathrm{CDCl}_{3}\right) \delta 1.31\left(\mathrm{t},{ }^{3} J_{\mathrm{HH}}=6.9,6 \mathrm{H}, \mathrm{OCH}_{2} \mathrm{CH}_{3}\right), 1.17-1.55(\mathrm{~m}$, $\left.4 \mathrm{H}, \mathrm{C}_{3} \mathrm{H}_{\mathrm{ax}}, \mathrm{C}_{4} \mathrm{H}_{\mathrm{ax}}, \mathrm{C}_{4} \mathrm{H}_{\mathrm{eq}}\right), 1.67-1.82\left(\mathrm{~m}, 2 \mathrm{H}, \mathrm{C}_{3} \mathrm{H}_{\mathrm{eq}}\right), 1.88-1.98\left(\mathrm{~m}, 2 \mathrm{H}, \mathrm{C}_{2} \mathrm{H}_{\mathrm{ax}}\right), 2.15-2.26(\mathrm{~m}, 2 \mathrm{H}$, $\left.\mathrm{C}_{2} \mathrm{H}_{\mathrm{eq}}\right), 4.02-4.16\left(\mathrm{~m}, 4 \mathrm{H}, \mathrm{OCH}_{2} \mathrm{CH}_{3}\right), 4.38-4.52\left(\mathrm{~m}, 1 \mathrm{H}, \mathrm{C}_{1} \mathrm{H}\right), 5.18\left(\mathrm{~d},{ }^{3} \mathrm{~J}_{\mathrm{HP}}=9.2,2 \mathrm{H}, \mathrm{CH}_{2} \mathrm{O}\right), 7.70$ $(\mathrm{s}, 1 \mathrm{H}, \mathrm{CH}) ;{ }^{13} \mathrm{C} \mathrm{NMR}\left(\mathrm{CDCl}_{3}\right) \delta 16.2\left(\mathrm{~d},{ }^{3} J_{\mathrm{CP}}=6.8, \mathrm{OCH}_{2} \mathrm{CH}_{3}\right), 25.2\left(\mathrm{C}_{4}\right), 25.3\left(\mathrm{C}_{3}\right), 33.7\left(\mathrm{C}_{2}\right), 60.3$ $\left(\mathrm{C}_{1}\right), 60.8\left(\mathrm{~d},{ }^{2} J_{\mathrm{CP}}=5.2, \mathrm{CH}_{2} \mathrm{O}\right), 64.0\left(\mathrm{~d},{ }^{2} J_{\mathrm{CP}}=5.9, \mathrm{OCH}_{2} \mathrm{CH}_{3}\right), 121.3(\mathrm{CH}=), 142.9\left(\mathrm{~d},{ }^{3} J_{\mathrm{CP}}=6.8, \mathrm{C}=\right)$; $[\mathrm{M}+\mathrm{H}]^{+}$found $=318.1575, \mathrm{C}_{13} \mathrm{H}_{25} \mathrm{~N}_{3} \mathrm{O}_{4} \mathrm{P}$ requires 318.1583 .

(1-Phenyl-1H-1,2,3-triazol-4-yl)methyl diethyl phosphate (14j): Yield: 73\% (0.23 g), pale yellow oil; ${ }^{31} \mathrm{P}$ NMR $\left(\mathrm{CDCl}_{3}\right) \delta-1.0 ;{ }^{1} \mathrm{H}$ NMR $\left(\mathrm{CDCl}_{3}\right) \delta 1.33\left(\mathrm{t},{ }^{3} \mathrm{~J}_{\mathrm{HH}}=7.1,6 \mathrm{H}, \mathrm{OCH}_{2} \mathrm{CH}_{3}\right), 3.99-4.20(\mathrm{~m}, 4 \mathrm{H}$, $\left.\mathrm{OCH}_{2} \mathrm{CH}_{3}\right), 5.28\left(\mathrm{~d},{ }^{3} J_{\mathrm{HP}}=9.2,2 \mathrm{H}, \mathrm{CH}_{2} \mathrm{O}\right), 7.37-7.61\left(\mathrm{~m}, 9 \mathrm{H}, \mathrm{C}_{3} \mathrm{H}, \mathrm{C}_{4} \mathrm{H}\right), 7.74\left(\mathrm{~d},{ }^{3} J_{\mathrm{HH}}=7.8,2 \mathrm{H}, \mathrm{C}_{2} \mathrm{H}\right)$, $8.16(\mathrm{~s}, 1 \mathrm{H}, \mathrm{CH}) ;{ }^{13} \mathrm{C}$ NMR $\left(\mathrm{CDCl}_{3}\right) \delta 16.2\left(\mathrm{~d},{ }^{3} J_{\mathrm{CP}}=6.8, \mathrm{OCH}_{2} \mathrm{CH}_{3}\right), 60.5\left(\mathrm{~d},{ }^{2} J_{\mathrm{CP}}=5.1, \mathrm{CH}_{2} \mathrm{O}\right), 64.2$ $\left(\mathrm{d},{ }^{2} J_{\mathrm{CP}}=6.0, \mathrm{OCH}_{2} \mathrm{CH}_{3}\right), 120.7\left(\mathrm{C}_{2}\right), 121.8(\mathrm{CH}=), 129.1\left(\mathrm{C}_{4}\right), 129.9\left(\mathrm{C}_{3}\right), 137.0\left(\mathrm{C}_{1}\right), 144.1\left(\mathrm{~d},{ }^{3} J_{\mathrm{CP}}=7.0\right.$, $\mathrm{C}=) ;[\mathrm{M}+\mathrm{H}]^{+}$found $=312.1104, \mathrm{C}_{13} \mathrm{H}_{19} \mathrm{~N}_{3} \mathrm{O}_{4} \mathrm{P}$ requires 312.1113 .

\section{Conclusions}

In summary, we have developed a facile, efficient method for the synthesis of new (1-alkyl/aryl-1H-1,2,3-triazol-4-yl)methyl phosphinates or (1-alkyl/aryl-1H-1,2,3-triazol-4-yl)methyl 
diethyl phosphates by the copper(I)-catalyzed azide-alkyne cycloaddition of organic azides and prop-2-ynyl phosphinate or diethyl prop-2-ynyl phosphate. This method, which has the advantages of simple operation and mild reaction conditions, is a novel approach for the synthesis of the target products. Altogether, 20 new derivatives were synthesized and fully characterized.

Supplementary Materials: Supplementary data associated with this article are available online. Copies of ${ }^{31} \mathrm{P}$, ${ }^{1} \mathrm{H}$, and ${ }^{13} \mathrm{C}$ NMR spectra for all compounds synthesized are presented.

Author Contributions: Conceptualization, E.B.; methodology, E.B. and A.T.; validation, K.N. and P.T.S.; formal analysis, K.N. and P.T.S.; investigation, A.T.; writing—original draft preparation, E.B.; writing — review and editing, A.T.; visualization, E.B. and A.T.; supervision, E.B.; project administration, A.T.; funding acquisition, E.B.

Funding: The project was supported by the Hungarian Research Development and Innovation Office (FK123961). E.B. was supported by the János Bolyai Research Scholarship of the Hungarian Academy of Sciences (BO/00278/17/7), and by the UNNKP-18-4-BME-131 New National Excellence Program of the Ministry of Human Capacities. A.T. was supported by the ÚNKP-18-3-I-BME-119 New National Excellence Program of the Ministry of Human Capacities.

Conflicts of Interest: The authors declare no conflict of interest.

\section{References}

1. Katritzky, A.R.; Rees, C.W.; Scriven, C.W.V. (Eds.) Comprehensive Heterocyclic Chemistry; Elsevier Science: Amsterdam, The Netherlands, 1996; Volume 4, pp. 1-126.

2. Thirumurugan, P.; Matosiuk, D.; Jozwiak, K. Click chemistry for drug development and diverse chemical-biology applications. Chem. Rev. 2013, 113, 4905-4979. [CrossRef] [PubMed]

3. Lauria, A.; Delisi, R.; Mingoia, F.; Terenzi, A.; Martorana, A.; Barone, G.; Almerico, A.M. 1,2,3-Triazole in heterocyclic compounds, endowed with biological activity, through 1,3-dipolar cycloadditions. Eur. J. Org. Chem. 2014, 3289-3306. [CrossRef]

4. Dheer, D.; Singh, V.; Shankar, R. Medicinal attributes of 1,2,3-triazoles: Current developments. Bioorg. Chem. 2017, 71, 30-54. [CrossRef] [PubMed]

5. Williams, A. Opportunities for chiral agrochemicals. Pestic. Sci. 1996, 46, 3-9. [CrossRef]

6. Ali, G.Q.; El-Hiti, G.A.; Tomi, I.H.; Haddad, R.; Al-Qaisi, A.J.; Yousif, E. Photostability and performance of polystyrene films containing 1,2,4-triazole-3-thiol ring system schiff bases. Molecules 2016, 21, 1699-1711. [CrossRef] [PubMed]

7. Bouchemella, K.; Fauché, K.; Anak, B.; Jouffret, L.; Bencharif, M.; Cisnetti, F. Click 1,2,3-triazole derived fluorescent scaffold by mesoionic carbene-nitrene cyclization: an experimental and theoretical study. New J. Chem. 2018, 42, 18969-18978. [CrossRef]

8. Srividhya, D.; Manjunathan, S.; Thirumaran, S.; Saravanan, C.; Senthil, S. Synthesis and characterization of [1,2,3]-triazole containing liquid crystals through click reaction. J. Mol. Struct. 2009, 927, 7-13. [CrossRef]

9. Tajti, Á.; Keglevich, G. The importance of organophosphorus compounds as biologically active agents. In Organophosphorus Chemistry; Keglevich, G., Ed.; Walter de Gruyter GmbH: Berlin, Germany, 2018; pp. 53-65. ISBN 978-3-11-053453-5.

10. Li, L.; Hao, G.; Zhu, A.; Fan, X.; Zhang, G.; Zhang, L. A copper(I)-catalyzed three-component domino process: assembly of complex 1,2,3-triazolyl-5-phosphonates from azides, alkynes, and H-phosphates. Chem. Eur. J. 2013, 19, 14403-14406. [CrossRef]

11. Kee, J.M.; Villani, B.; Carpenter, L.R.; Muir, T.W. Development of stable phosphohistidine analogues. J. Am. Chem. Soc. 2010, 132, 14327-14329. [CrossRef]

12. Mukai, S.; Flematti, G.R.; Byrne, L.T.; Besant, P.G.; Attwood, P.V.; Piggott, M.J. Stable triazolylphosphonate analogues of phosphohistidine. Amino Acids 2012, 43, 857-874. [CrossRef]

13. Radi, S.; Lazrek, H.B. Synthesis and biological activity of new 1,2,3-triazole acyclonucleosides analogues of ACV. J. Chem. Res. Synop. 2002, 264-266. [CrossRef]

14. Rostovtsev, V.V.; Green, L.G.; Fokin, V.V.; Sharpless, K.B. A stepwise huisgen cycloaddition process: Copper(I)-catalyzed regioselective "ligation" of azides and terminal alkynes. Angew. Chem. Int. Ed. 2002, 41, 2596-2599. [CrossRef] 
15. Tornøe, C.W.; Christensen, C.; Meldal, M. Peptidotriazoles on solid phase: [1,2,3]-Triazoles by regiospecific copper(I)-catalyzed 1,3-dipolar cycloadditions of terminal alkynes to azides. J. Org. Chem. 2002, 67,3057-3064. [CrossRef] [PubMed]

16. Thiery, E.; You, V.; Mora, A.-S.; Abarbri, M. Synthesis of 5-substituted 1,2,3-triazolyl-4-phosphonate through cross-coupling reactions of 5-iodo-1,2,3-triazolyl-4-phosphonate. Eur. J. Org. Chem. 2016, 529-534. [CrossRef]

17. Skarpos, H.; Osipov, S.N.; Vorob'eva, D.V.; Odinets, I.L.; Lork, E.; Roschenthaler, G.V. Synthesis of functionalized bisphosphonates via click chemistry. Org. Biomol. Chem. 2007, 5, 2361-2367. [CrossRef] [PubMed]

18. Zhou, X.; Hartman, S.V.; Born, E.J.; Smits, J.P.; Holstein, S.A.; Wiemer, D.F. Triazole-based inhibitors of geranylgeranyltransferase II. Bioorg. Med. Chem. Lett. 2013, 23, 764-766. [CrossRef] [PubMed]

19. Vorobyeva, D.V.; Karimova, N.M.; Vasilyeva, T.P.; Osipov, S.N.; Shchetnikov, G.T.; Odinets, I.L.; Röschenthaler, G.-V. Synthesis of functionalized $\alpha$-CF3- $\alpha$-aminophosphonates via $\mathrm{Cu}(\mathrm{I})$-catalyzed 1,3-dipolar cycloaddition. J. Fluorine Chem. 2010, 131, 378-383. [CrossRef]

20. Sykam, K.; Meka, K.K.R.; Donempudi, S. Intumescent phosphorus and triazole-based flame-retardant polyurethane foams from castor oil. ACS Omega 2019, 4, 1086-1094. [CrossRef]

21. Artyushin, O.I.; Vorob'eva, D.V.; Vasil'eva, T.P.; Osipov, S.N.; Röschenthaler, G.-V.; Odinets, I.L. Facile synthesis of phosphorylated azides in ionic liquids and their use in the preparation of 1,2,3-triazoles. Heteroat. Chem. 2008, 19, 293-300. [CrossRef]

22. Artyushin, O.I.; Matveeva, E.V.; Bushmarinov, I.S.; Odinets, I.L. Water as a promoting media for 1,3-dipolar cycloaddition of phosphorylated azides to internal alkynes. Arkivoc 2012, iv, 252-263. [CrossRef]

23. Glowacka, I.E.; Balzarini, J.; Wroblewski, A.E. Design, synthesis, antiviral, and cytotoxic evaluation of novel phosphonylated 1,2,3-triazoles as acyclic nucleotide analogues. Nucleosides Nucleotides Nucleic Acids 2012, 31, 293-318. [CrossRef] [PubMed]

24. Veliscek-Carolan, J.; Rawal, A. Zirconium bistriazolylpyridine phosphonate materials for efficient, selective An(iii)/Ln(iii) separations. Chem. Commun. 2019, 55, 1168-1171. [CrossRef] [PubMed]

25. Erzunov, D.A.; Latyshev, G.V.; Averin, A.D.; Beletskaya, I.P.; Lukashev, N.V. CuAAC synthesis and anion binding properties of bile acid derived tripodal ligands. Eur. J. Org. Chem. 2015, 6289-6297. [CrossRef]

26. Gann, A.W.; Amoroso, J.W.; Einck, V.J.; Rice, W.P.; Chambers, J.J.; Schnarr, N.A. A photoinduced, benzyne click reaction. Org. Lett. 2014, 16, 2003-2005. [CrossRef] [PubMed]

27. Womble, C.T.; Coates, G.W.; Matyjaszewski, K.; Noonan, K.J.T. Tetrakis(dialkylamino)phosphonium polyelectrolytes prepared by reversible addition-fragmentation chain transfer polymerization. ACS Macro Lett. 2016, 5, 253-257. [CrossRef]

28. Bao, M.; Lu, W.; Su, H.; Qiu, L.; Xu, X. A convergent formal [4 + 2] cycloaddition of 1,6-diynes and benzyl azides: construction ofspiro-polyheterocycles. Org. Biomol. Chem. 2018, 16, 3258-3265. [CrossRef]

29. Wang, X.; Mei, T.-S.; Yu, J.-Q. Versatile $\mathrm{Pd}(\mathrm{OTf})_{2} \cdot 2 \mathrm{H}_{2} \mathrm{O}$-catalyzed ortho-fluorination using NMP as a promoter. J. Am. Chem. Soc. 2009, 131, 7520-7521. [CrossRef]

30. Colombano, G.; Albani, C.; Ottonello, G.; Ribeiro, A.; Scarpelli, R.; Tarozzo, G.; Daglian, J.; Jung, K.-M.; Piomelli, D.; Bandiera, T. O-(Triazolyl)methyl carbamates as a novel and potent class of fatty acid amide gydrolase (FAAH) inhibitors. Chem. Med. Chem. 2014, 10, 380-395. [CrossRef]

31. Tesch, M.; Kudruk, S.; Letzel, M.; Studer, A. Orthogonal click postfunctionalization of alternating copolymers prepared by nitroxide-mediated polymerization. Chem. Eur. J. 2017, 23, 5915-5919. [CrossRef]

32. Colombano, G.; Travelli, C.; Galli, U.; Caldarelli, A.; Chini, M.G.; Canonico, P.L.; Sorba, G.; Bifulco, G.; Tron, G.C.; Genazzani, A.A. A novel potent nicotinamide phosphoribosyltransferase inhibitor synthesized via click chemistry. J. Med. Chem. 2010, 53, 616-623. [CrossRef]

33. Swetha, M.; Ramana, P.V.; Shirodkar, S.G. Simple and efficient method for the synthesis of azides in water-THF solvent system. Org. Prep. Proced. Int. 2011, 43, 348-353. [CrossRef]

34. Jones, S.; Smanmoo, C. Phosphorylation of alcohols with $N$-phosphoryl oxazolidinones employing copper(II) triflate catalysis. Org. Lett. 2005, 7, 3271-3274. [CrossRef] [PubMed]

(C) 2019 by the authors. Licensee MDPI, Basel, Switzerland. This article is an open access article distributed under the terms and conditions of the Creative Commons Attribution (CC BY) license (http://creativecommons.org/licenses/by/4.0/). 